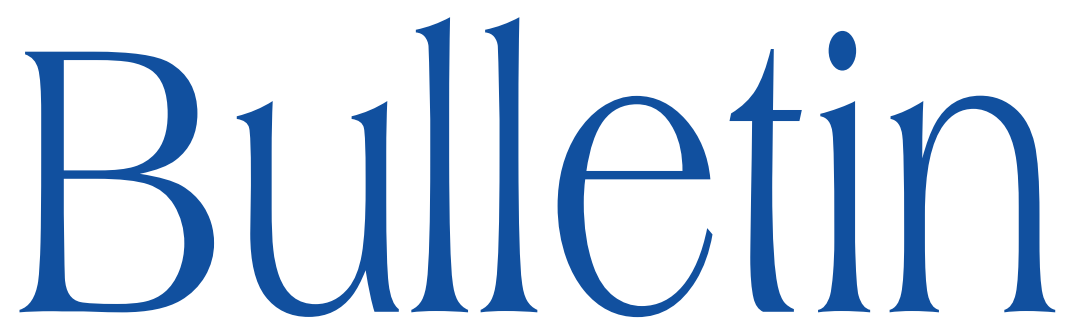

de la SOCIÉTÉ MATHÉMATIQUE DE FRANCE

\title{
THE DIXMIER-MOEGLIN EQUIVALENCE AND A GEL'FAND-KIRILLOV PROBLEM FOR POISSON POLYNOMIAL ALGEBRAS
}

Tome $139^{\text {K. R. Goodearl \& S. Launois }}$ Fascicule 1

2011 
Bull. Soc. math. France

139 (1), 2011, p. 1-39

\title{
THE DIXMIER-MOEGLIN EQUIVALENCE AND A GEL'FAND-KIRILLOV PROBLEM FOR POISSON POLYNOMIAL ALGEBRAS
}

\author{
By K. R. Goodearl \& S. Launois
}

\begin{abstract}
The structure of Poisson polynomial algebras of the type obtained as semiclassical limits of quantized coordinate rings is investigated. Sufficient conditions for a rational Poisson action of a torus on such an algebra to leave only finitely many Poisson prime ideals invariant are obtained. Combined with previous work of the first-named author, this establishes the Poisson Dixmier-Moeglin equivalence for large classes of Poisson polynomial rings, including semiclassical limits of quantum matrices, quantum symplectic and euclidean spaces, quantum symmetric and antisymmetric matrices. For a similarly large class of Poisson polynomial rings, it is proved that the quotient field of the algebra (respectively, of any Poisson prime factor ring) is a rational function field $F\left(x_{1}, \ldots, x_{n}\right)$ over the base field (respectively, over an extension field of the base field) with $\left\{x_{i}, x_{j}\right\}=\lambda_{i j} x_{i} x_{j}$ for suitable scalars $\lambda_{i j}$, thus establishing a quadratic Poisson version of the Gel'fand-Kirillov problem. Finally, partial solutions to the isomorphism problem for Poisson fields of the type just mentioned are obtained.
\end{abstract}

Texte reçu le 12 juin 2007, révisé le 17 décembre 2009, accepté le 14 janvier 2010

K. R. Goodearl, Department of Mathematics, University of California, Santa Barbara, CA 93106, USA - E-mail : goodearl@math.ucsb.edu

S. Launors, School of Mathematics, Statistics and Actuarial Science, University of Kent at Canterbury, CT2 7NF, UK • E-mail : S.Launois@kent.ac.uk

2000 Mathematics Subject Classification. - 17B63.

Key words and phrases. - Poisson polynomial algebras, Dixmier-Moeglin equivalence, Gel'fand-Kirillov problem.

This research was partially supported by Leverhulme Research Interchange Grant F/00158/X (UK), that of the first-named author by a grant from the National Science Foundation (USA), and that of the second-named author by a Marie Curie Intra-European Fellowship held at the University of Edinburgh within the $6^{\text {th }}$ European Community Framework Programme and by a Marie Curie Reintegration Grant within the $7^{\text {th }}$ European Community Framework Programme. 
RÉsumé (L'équivalence de Dixmier-Moeglin et un analogue du problème de Gel'fandKirillov pour certaines algèbres de Poisson polynômiales)

Nous étudions la structure de certaines algèbres de Poisson polynômiales obtenues comme limites semi-classiques de certaines déformations quantiques d'anneaux de fonctions régulières. Lorsqu'un tore agit rationnellement sur une telle algèbre de Poisson, nous donnons une condition suffisante pour que cette algèbre n'ait qu'un nombre fini d'idéaux premiers de Poisson invariants sous cette action. Ce résultat, combiné à des résultats antérieurs de K.R. Goodearl, permet d'établir l'équivalence de Dixmier-Moeglin pour une large classe d'algèbres de Poisson polynômiales incluant les limites semi-classiques des matrices quantiques, des espaces Euclidiens and symplectiques quantiques, des matrices symétriques et antisymétriques quantiques. De plus, nous démontrons que le corps des fractions de ces algèbres (respectivement, de leurs quotients premiers de Poisson) est un corps de fractions rationnelles $F\left(x_{1}, \ldots, x_{n}\right)$ sur le corps de base (respectivement, sur une certaine extension du corps de base) dont la structure de Poisson est de la forme $\left\{x_{i}, x_{j}\right\}=\lambda_{i j} x_{i} x_{j}$ pour certains scalaires $\lambda_{i j}$ convenablement choisis. Ce résultat est un analogue quadratique du problème de Gel'fand-Kirillov pour la structure de Poisson de ces corps. Finallement, nous présentons des résultat partiels quant à la classification de tels corps de fractions à isomorphisme (de Poisson) près.

\section{Introduction}

Many properties of the noncommutative algebras appearing in the world of quantum groups are known, or are conjectured to be, reflected in parallel properties of the Poisson algebras that arise as their semiclassical limits. The present work targets two fundamental properties - the Dixmier-Moeglin equivalence and the quantum Gel'fand-Kirillov conjecture - and establishes Poisson versions of them for large classes of Poisson algebras of the type appearing as semiclassical limits of quantized coordinate rings. More detail follows.

Fix a base field $k$ of characteristic zero throughout. All algebras are assumed to be over $k$, and all relevant maps (automorphisms, derivations, etc.) are assumed to be $k$-linear.

0.1. Poisson algebras. - Recall that a Poisson algebra (over $k$ ) is a commutative $k$-algebra $A$ equipped with a Lie bracket $\{-,-\}$ which is a derivation (for the associative multiplication) in each variable. We investigate (iterated) Poisson polynomial algebras over $k$, that is, polynomial algebras $k\left[x_{1}, \ldots, x_{n}\right]$ equipped with Poisson brackets such that

$$
\left\{x_{i}, k\left[x_{1}, \ldots, x_{i-1}\right]\right\} \subseteq k\left[x_{1}, \ldots, x_{i-1}\right] x_{i}+k\left[x_{1}, \ldots, x_{i-1}\right]
$$

for $i=2, \ldots, n$ (see $\S 1.1$ for more detail on the conditions satisfied by such a bracket). Many such Poisson algebras are semiclassical limits of quantum algebras, and these provide our motivation and focus (see Section 2). The 
Kirillov-Kostant-Souriau Poisson structure on the symmetric algebra of a finite dimensional Lie algebra $\mathfrak{g}$ can be put in the form of a Poisson polynomial algebra when $\mathfrak{g}$ is completely solvable. This also holds for the basic example of a Poisson-Weyl algebra, namely a polynomial algebra $k\left[x_{1}, \ldots, x_{n}, y_{1}, \ldots, y_{n}\right]$ equipped with the Poisson bracket such that

$$
\left\{x_{i}, x_{j}\right\}=\left\{y_{i}, y_{j}\right\}=0 \quad\left\{x_{i}, y_{j}\right\}=\delta_{i j}
$$

for all $i, j$.

0.2. The Dixmier-Moeglin equivalence. - Let $A$ be a noetherian algebra over a field $k$ (here positive characteristic is allowed). As a first approximation to the (usually complicated) representation theory of $A$, Dixmier raised the question of classifying the primitive ideals of $A$, that is, the annihilators of simple (left) modules over $A$. In general, one expects to characterize the primitive ideals of $A$ among its prime ideals either topologically or algebraically as follows. A prime ideal $P$ of $A$ is locally closed provided $P$ is a locally closed point of the prime spectrum $\operatorname{spec} A$, and $P$ is rational if the center of the Goldie quotient ring Fract $A / P$ is algebraic over $k$. One says that the Dixmier-Moeglin equivalence holds in $A$ provided the sets of primitive ideals, locally closed prime ideals, and rational prime ideals coincide. This equivalence was first proved by Dixmier [7] and Moeglin [22] for enveloping algebras of finite dimensional complex Lie algebras, and then extended to arbitrary base fields of characteristic zero by Irving and Small [16]. For quantized coordinate rings of semisimple groups, it follows from work of Hodges, Levasseur, Joseph, and Toro [12, 13, 14, 17, 18] (see $[10, \S 2.4]$ for details). It was established for large classes of other quantized coordinate rings, including the ones of interest in the present work, by Letzter and the first-named author [10] (see also [2, Chapter II.8]).

0.3. The Poisson Dixmier-Moeglin equivalence. - Let $A$ be a Poisson algebra. A Poisson ideal of $A$ is any ideal $I$ such that $\{A, I\} \subseteq I$, and a Poisson prime ideal is any prime ideal which is also a Poisson ideal. The set of Poisson prime ideals in $A$ forms the Poisson prime spectrum, denoted P.spec $A$, which is given the relative Zariski topology inherited from $\operatorname{spec} A$. Given an arbitrary ideal $J$ of $A$, there is a largest Poisson ideal contained in $J$, called the Poisson core of $J$. The Poisson-primitive ideals of $A$ are the Poisson cores of the maximal ideals. (One thinks of the Poisson core of an ideal in a Poisson algebra as analogous to the bound of a left ideal $L$ in a noncommutative algebra $B$, that is, the largest two-sided ideal of $B$ contained in $L$.) The Poisson-primitive ideals in the coordinate ring of a complex affine Poisson variety $V$ are the defining ideals of the Zariski closures of the symplectic leaves in $V$ [3, Lemma 3.5], and they are the key to Brown and Gordon's concept of symplectic cores [3, §3.3]. 
The Poisson center of $A$ is the subalgebra

$$
Z_{p}(A)=\{z \in A \mid\{z,-\} \equiv 0\} .
$$

For any Poisson prime ideal $P$ of $A$, there is an induced Poisson bracket on $A / P$, which extends uniquely to the quotient field Fract $A / P$ (e.g., [21, Proposition 1.7]). We say that $P$ is Poisson rational if the field $Z_{p}($ Fract $A / P)$ is algebraic over $k$.

By analogy with the Dixmier-Moeglin equivalence, we say that $A$ satisfies the Poisson Dixmier-Moeglin equivalence (e.g., [27, pp. 7,8]) provided the following sets coincide:

1. The set of Poisson-primitive ideals in $A$;

2. The set of locally closed points in P.spec $A$;

3. the set of Poisson rational Poisson prime ideals of $A$.

If $A$ is an affine (i.e., finitely generated) $k$-algebra, then $(2) \subseteq(1) \subseteq(3)[27$, Propositions $1.7,1.10$ ], so the main difficulty is whether $(3) \subseteq(2)$. No examples are known of affine Poisson algebras for which the Poisson Dixmier-Moeglin equivalence fails.

We obtain this equivalence for Poisson polynomial algebras via the main result of [8], which established it for Poisson algebras with suitable torus actions, as follows.

0.4. Torus actions. - Suppose that $H$ is a group acting on a Poisson algebra $A$ by Poisson automorphisms (i.e., $k$-algebra automorphisms that preserve the Poisson bracket). For each $H$-stable Poisson prime $J$ of $A$, set

$$
\text { P.spec }_{J} A=\left\{P \in \text { P.spec } A \mid \bigcap_{h \in H} h(P)=J\right\},
$$

the $H$-stratum of P.spec $A$ corresponding to $J$. These $H$-strata partition P.spec $A$ as $J$ runs through the $H$-stable Poisson primes of $A$.

Now assume that $H=\left(k^{\times}\right)^{r}$ is an algebraic torus over $k$. In this case, the action of $H$ on $A$ is called rational provided $A$ is generated (as a $k$-algebra) by $H$-eigenvectors whose eigenvalues are rational characters of $A$. (See $\S 1.2$ for the general definition of a rational action of an algebraic group, and [2, Theorem II.2.7] for the equivalence with the above condition in the case of a torus.) In this situation, the Poisson Dixmier-Moeglin equivalence holds when the number of $H$-stable Poisson prime ideals in $A$ is finite. More precisely, the following result was proved in [8].

Theorem 0.1. - [8, Theorem 4.3] Let $A$ be an affine Poisson algebra and $H=\left(k^{\times}\right)^{r}$ an algebraic torus acting rationally on $A$ by Poisson automorphisms. Assume that $A$ has only finitely many $H$-stable Poisson prime ideals. 
Then the Poisson Dixmier-Moeglin equivalence holds in A, and the Poisson primitive ideals are precisely those Poisson primes maximal in their H-strata.

Most of the examples that we will consider in this paper will support a useful rational torus action by Poisson automorphisms. In view of the above theorem, all we will need to establish the Poisson Dixmier-Moeglin equivalence is that the number of $H$-stable Poisson prime ideals is finite in these examples. In the first section, we establish a criterion for a Poisson algebra endowed with a rational torus action to have only finitely many $H$-stable Poisson primes. This criterion applies in particular to semiclassical limits of many quantized coordinate rings, as summarized in the next theorem. The Poisson Dixmier-Moeglin equivalence was already known in some of these cases due to work of Oh [27, 28] and the first-named author [8]. However, in the present text, we give a uniform proof and get new examples of Poisson algebras for which this equivalence holds.

Theorem 0.2. - Let the Poisson algebra $A$ be a semiclassical limit of quantum affine spaces, quantum matrices, quantum symplectic or euclidean spaces, quantum symmetric or antisymmetric matrices, equipped with the natural ("standard") rational Poisson action of an algebraic torus $H$, as detailed in Section 2.

Then there are only finitely many $H$-stable Poisson prime ideals in $A$, and, consequently, the Poisson Dixmier-Moeglin equivalence holds in A.

0.5. The quantum Gel'fand-Kirillov problem. - The original Gel'fand-Kirillov problem asked whether the quotient division ring of the enveloping algebra of a finite dimensional algebraic Lie algebra over $k$ is isomorphic to a Weyl skew field over a purely transcendental extension $K$ of $k$, i.e., the quotient division ring of a Weyl algebra over $K$.

Quantum versions of the Gel'fand-Kirillov problem have been studied by a number of authors (e.g., see [2, pp. 230-231] for a summary). These involve quotient division rings of quantized Weyl algebras, which turn out to be isomorphic to quotient division rings of quantum affine spaces

$$
\left.\vartheta_{\mathbf{q}}\left(k^{n}\right)=k\left\langle x_{1}, \ldots, x_{n}\right| x_{i} x_{j}=q_{i j} x_{j} x_{i} \text { for all } i, j\right\rangle
$$

for multiplicatively antisymmetric matrices $\mathbf{q}=\left(q_{i j}\right) \in M_{n}\left(k^{\times}\right)$. Thus, one asks whether the quotient division ring of a quantized algebra $A$, or of any prime factor of $A$, must be isomorphic to Fract $\theta_{\mathbf{q}}\left(K^{n}\right)$ for suitable $K, \mathbf{q}, n$. 
0.6. Poisson Gel'fand-Kirillov problems. - Vergne raised the Poisson version of the Gel'fand Kirillov question for the Kirillov-Kostant-Souriau Poisson structure on the symmetric algebra of a Lie algebra $\mathfrak{g}$, namely whether the quotient field of $S(\mathfrak{g})$ is isomorphic (as a Poisson algebra) to the quotient field of a Poisson-Weyl algebra [36, Introduction], and answered this positively for nilpotent $\mathfrak{g}$ [36, Théorème 4.1]. We shall use the term Poisson-Weyl field for the quotient field of a Poisson-Weyl algebra, that is, for a rational function field $K\left(x_{1}, \ldots, x_{n}, y_{1}, \ldots, y_{n}\right)$ equipped with the (unique) $K$-linear Poisson bracket satisfying (1). Vergne's result was extended to algebraic solvable Lie algebras $\mathfrak{g}$, and to Poisson prime factors of $S(\mathfrak{g})$ for such $\mathfrak{g}$, by Tauvel and Yu [34, Corollaire 11.8]. We also mention that Kostant and Wallach showed that a Galois extension of the quotient field of $\Theta\left(M_{n}(\mathbb{C})\right)$, with a natural Poisson structure, is a Poisson-Weyl field [20, Theorem 5.24].

The above form of a Poisson Gel'fand-Kirillov problem is not appropriate for the algebras we consider. In fact, as we prove in Corollary 5.3, the quotient field of a semiclassical limit of a typical quantum algebra can never be isomorphic to a Poisson-Weyl field. A suitable version is suggested by looking at semiclassical limits of quantum affine spaces, which are Poisson polynomial rings $k\left[x_{1}, \ldots, x_{n}\right]$ with Poisson brackets satisfying

$$
\left\{x_{i}, x_{j}\right\}=\lambda_{i j} x_{i} x_{j}
$$

for all $i, j$, where $\boldsymbol{\lambda}=\left(\lambda_{i j}\right)$ is an antisymmetric $n \times n$ matrix over $k$ (see $\left.\S 2.2\right)$. It will be convenient to denote this Poisson polynomial algebra by $k_{\boldsymbol{\lambda}}\left[x_{1}, \ldots, x_{n}\right]$, the corresponding Poisson Laurent polynomial ring by $k_{\boldsymbol{\lambda}}\left[x_{1}^{ \pm 1}, \ldots, x_{n}^{ \pm 1}\right]$, and the corresponding Poisson field by $k_{\boldsymbol{\lambda}}\left(x_{1}, \ldots, x_{n}\right)$. In all three cases, the Poisson bracket is uniquely determined by (2), for instance because Poisson brackets extend uniquely to localizations [21, Proposition 1.7]. In the present situation, however, the extensions are easier to establish, since we can give them by the formula

$$
\{f, g\}=\sum_{i, j=1}^{n} \lambda_{i j} x_{i} x_{j} \frac{\partial f}{\partial x_{i}} \frac{\partial g}{\partial x_{j}}
$$

For semiclassical limits of quantum algebras, a natural version of the Gel'fand-Kirillov problem is thus to ask whether the quotient field is isomorphic to a Poisson field of the form $k_{\boldsymbol{\lambda}}\left(x_{1}, \ldots, x_{n}\right)$, or at least $K_{\boldsymbol{\lambda}}\left(x_{1}, \ldots, x_{n}\right)$ where $K$ is an extension field of $k$. We establish the former for large classes of Poisson polynomial algebras, and the latter for Poisson prime factors of these algebras, in particular obtaining the theorem below. 
In the last section of the paper, we introduce some invariants for Poisson fields, with which we show that $k_{\boldsymbol{\lambda}}\left(x_{1}, \ldots, x_{n}\right)$ is never isomorphic to a PoissonWeyl field, and with which we can separate isomorphism classes of the Poisson fields $k_{\boldsymbol{\lambda}}\left(x_{1}, \ldots, x_{n}\right)$ in many cases.

Theorem 0.3. - Let the Poisson algebra $A$ be a semiclassical limit of quantum affine spaces, quantum matrices, quantum symplectic or euclidean spaces, quantum symmetric or antisymmetric matrices, and let $P$ be any Poisson prime ideal of $A$. Then there exist a field extension $K \supseteq k$ and an antisymmetric matrix $\boldsymbol{\mu} \in M_{m}(k)$, for some $m$, such that Fract $A / P \cong K_{\boldsymbol{\mu}}\left(y_{1}, \ldots, y_{m}\right)$ (as Poisson algebras). In case $P=0$, we have $K=k$ and $m=\operatorname{tr} \cdot \operatorname{deg}_{k} A$.

\section{A finiteness theorem for torus-stable Poisson primes}

In this section, we prove our finiteness theorem for the number of Poisson prime ideals stable under a suitable torus action on an iterated Poisson polynomial algebra. We begin by recalling the concept of a Poisson polynomial algebra as introduced by $\mathrm{Oh}[28]$.

1.1. Poisson polynomial algebras. - Let $B$ be a Poisson algebra. A Poisson derivation on $B$ is a ( $k$-linear) map $\alpha$ on $B$ which is a derivation with respect to both the multiplication and the Poisson bracket, that is, $\alpha(a b)=\alpha(a) b+a \alpha(b)$ and $\alpha(\{a, b\})=\{\alpha(a), b\}+\{a, \alpha(b)\}$ for $a, b \in B$. Suppose that $\delta$ is a derivation on $B$ such that

$$
\delta(\{a, b\})=\{\delta(a), b\}+\{a, \delta(b)\}+\alpha(a) \delta(b)-\delta(a) \alpha(b)
$$

for $a, b \in B$. By [28, Theorem 1.1] (after replacing our $B$ and $\alpha$ with $A$ and $-\alpha$ ), the Poisson structure on $B$ extends uniquely to a Poisson algebra structure on the polynomial ring $A=B[x]$ such that

$$
\{x, b\}=\alpha(b) x+\delta(b)
$$

for $b \in B$. We write $A=B[x ; \alpha, \delta]_{p}$ to denote this situation, and we refer to $A$ as a Poisson polynomial algebra.

The Poisson structure on $A$ extends uniquely to the Laurent polynomial ring $B\left[x^{ \pm 1}\right]$, and is again determined by $\alpha$ and $\delta$. Hence, we write $B\left[x^{ \pm 1} ; \alpha, \delta\right]_{p}$ for the ring $B\left[x^{ \pm 1}\right]$ equipped with this structure, and we refer to it as a Poisson Laurent polynomial algebra.

In either of the above cases, we omit $\delta$ from the notation if it is zero, that is, we write $B[x ; \alpha]_{p}$ and $B\left[x^{ \pm 1} ; \alpha\right]_{p}$ for $B[x ; \alpha, 0]_{p}$ and $B\left[x^{ \pm 1} ; \alpha, 0\right]_{p}$ respectively.

We will also need the converse part of [28, Theorem 1.1]: if a polynomial ring $A=B[x]$ supports a Poisson bracket such that $B$ is a Poisson subalgebra and $\{x, B\} \subseteq B x+B$, then $A=B[x ; \alpha, \delta]_{p}$ for suitable $\alpha$ and $\delta$. 
Lemma 1.1. - Let $A=B\left[x^{ \pm 1} ; \alpha\right]_{p}$ be a Poisson Laurent polynomial algebra, and assume that $\alpha$ extends to a derivation $\widehat{\alpha}$ on $A$ such that $\widehat{\alpha}(x)=$ sx for some nonzero $s \in k$. Then every $\widehat{\alpha}$-stable Poisson prime of $A$ is induced from a Poisson prime of $B$.

Proof. - Let $P$ be an $\widehat{\alpha}$-stable Poisson prime of $A$, and note that $P \cap B$ is a Poisson prime of $B$. Then $(P \cap B)\left[x^{ \pm 1}\right]$ is an $\widehat{\alpha}$-stable Poisson prime of $A$, and we may pass to $A /(P \cap B)\left[x^{ \pm 1}\right]$. Thus, without loss of generality, $P \cap B=0$, and we must show that $P=0$.

If $P \neq 0$, then $P \cap B[x] \neq 0$. Choose a nonzero polynomial $p \in P \cap B[x]$ of minimal degree, say $p=b_{n} x^{n}+b_{n-1} x^{n-1}+\cdots+b_{1} x+b_{0}$ with the $b_{i} \in B$ and $b_{n} \neq 0$. Note that $n>0$, because $P \cap B=0$. Now $P$ contains the polynomial

$$
\widehat{\alpha}(p)-\{x, p\} x^{-1}=n s b_{n} x^{n}+(n-1) s b_{n-1} x^{n-1}+\cdots+s b_{1} x,
$$

and hence also the polynomial

$$
n s p-\left(\widehat{\alpha}(p)-\{x, p\} x^{-1}\right)=s b_{n-1} x^{n-1}+\cdots+(n-1) s b_{1} x+n s b_{0} .
$$

The latter must vanish, due to the minimality of $n$, and so $b_{i}=0$ for $i<n$. But then $b_{n} x^{n}=p \in P$ and so $b_{n} \in P$, contradicting the assumption that $P \cap B=0$. Therefore $P=0$, as required.

Proposition 1.2. - Let $A=B[x ; \alpha, \delta]_{p}$ be a Poisson polynomial algebra, and assume that $\alpha$ extends to a derivation $\widehat{\alpha}$ on $A$ such that $\widehat{\alpha}(x)=s x$ for some nonzero $s \in k$. For each Poisson prime $Q$ of $B$, there are at most two $\widehat{\alpha}$-stable Poisson primes of $A$ that contract to $Q$.

Proof. - Assume there exists an $\widehat{\alpha}$-stable Poisson prime $P$ in $A$ that contracts to $Q$. For $b \in Q$, we have $\{x, b\} \in P$ and $\alpha(b)=\widehat{\alpha}(b) \in P$, whence $\delta(b)=$ $\{x, b\}-\alpha(b) x \in P$, and so $\alpha(b), \delta(b) \in Q$. It follows that $\{x, Q[x]\} \subseteq Q[x]$, from which we see that $Q[x]$ is an $\widehat{\alpha}$-stable Poisson prime of $A$. Hence, we may pass to $A / Q[x]$ and then localize $B / Q$ to its quotient field. Thus, without loss of generality, $B$ is a field, and we must show that $A$ has at most two $\widehat{\alpha}$-stable Poisson primes.

Assume there exists a nonzero $\widehat{\alpha}$-stable Poisson prime $P$ in $A$. Let $n$ be the minimum degree of nonzero elements of $P$, and choose a monic polynomial $p \in P$ of degree $n$, say $p=x^{n}+b_{n-1} x^{n-1}+\cdots+b_{1} x+b_{0}$ with the $b_{i} \in B$. Now $P$ contains the polynomial

$\widehat{\alpha}(p)-n s p=\left[\alpha\left(b_{n-1}\right)-s b_{n-1}\right] x^{n-1}+\cdots+\left[\alpha\left(b_{1}\right)-(n-1) s b_{1}\right] x+\left[\alpha\left(b_{0}\right)-n s b_{0}\right]$, 
which must be zero by the minimality of $n$, and so $\alpha\left(b_{n-1}\right)=s b_{n-1}$. For any $b \in B$, the following polynomial lies in $P$ :

$$
\begin{aligned}
\{p, b\}-n \alpha(b) p=\left[n \delta(b)+\left\{b_{n-1}, b\right\}+(n-1) b_{n-1} \alpha(b)-\right. & \left.n \alpha(b) b_{n-1}\right] x^{n-1} \\
+ & {[\text { lower terms }] }
\end{aligned}
$$

This polynomial must be zero, and so $n \delta(b)+\left\{b_{n-1}, b\right\}=\alpha(b) b_{n-1}$. Thus, the element $d:=\frac{1}{n} b_{n-1} \in B$ satisfies $\alpha(d)=s d$ and $\{d, b\}=\alpha(b) d-\delta(b)$ for $b \in B$.

Set $y:=x+d$. Then $A$ is a Poisson polynomial algebra of the form $A=$ $B[y ; \alpha]_{p}$. Further, $\widehat{\alpha}(y)=s y$, and so Lemma 1.1 implies that the only $\widehat{\alpha}$-stable Poisson prime of $B\left[y^{ \pm 1} ; \alpha\right]_{p}$ is zero. Therefore, the only $\widehat{\alpha}$-stable Poisson primes of $A$ are $\langle 0\rangle$ and $\langle y\rangle$.

Our finiteness theorem parallels a corresponding finiteness result of Letzter and the first author [10, Theorem 4.7], which applies to torus actions on iterated skew polynomial algebras. A key hypothesis in the latter theorem is that the automorphisms involved in the skew polynomial structure must be restrictions of elements of the acting torus. In the Poisson case, the corresponding ingredients are Poisson derivations, and the relevant hypothesis relates these to the differential of the torus action. We next recall the key facts about differentials of actions.

1.2. The differential of a group action. - Let $A$ be a $k$-algebra and $G$ an algebraic group over $k$, with Lie algebra $\mathfrak{g}$. Let $\alpha: G \rightarrow \operatorname{Aut}_{k \text {-alg }}(A)$ be a rational action of $G$ on a $k$-algebra $A$ by $k$-algebra automorphisms. Thus, $A$ is a directed union of finite dimensional $G$-stable subspaces $V_{i}$ such that the induced maps $\alpha_{i}: G \rightarrow G L\left(V_{i}\right)$ are morphisms of algebraic groups. In this situation, the following hold:

1. The differentials $d \alpha_{i}: \mathfrak{g} \rightarrow \mathfrak{g l}\left(V_{i}\right)$ are compatible with inclusions $V_{i} \subseteq V_{j}$, and they induce an action $d \alpha: \mathfrak{g} \rightarrow \operatorname{Der}_{k}(A)$.

2. If $G$ is connected, the $G$-stable subspaces of $A$ coincide with the $\mathfrak{g}$-stable subspaces.

That the $d \alpha_{i}$ are compatible with inclusions $V_{i} \subseteq V_{j}$ is a routine check, as in [33, Proposition 23.4.17]. One thus obtains a linear action $d \alpha: \mathfrak{g} \rightarrow \operatorname{End}_{k}(A)$, called the differential of the G-action. Statement (2) is proved in [33, Corollary 24.3.3] for the case that $k$ is algebraically closed, but the latter hypothesis is not required. The remainder of statement (1) is standard, but we have not located a precise reference. It can be quickly obtained from two results in [33], as follows. For each $i$, the multiplication map $V_{i} \otimes V_{i} \rightarrow V_{i}^{2} \subseteq A$ is $G$-equivariant, and so it is g-equivariant [33, Proposition 23.4.17]. Since the $G$-action on $V_{i} \otimes V_{i}$ is the diagonal one, so is the $\mathfrak{g}$-action [33, Proposition 23.4.12], from which we conclude that $\mathfrak{g}$ acts on $A$ by derivations. 
If $G$ is a torus, rationality of the action means that $A$ is the direct sum of its $G$-eigenspaces, and the corresponding eigenvalues are rational characters of $G$ (e.g., see [2, Theorem II.2.7]). In this case, $A$ is also the direct sum of its $\mathfrak{g}$-eigenspaces, and we have the following explicit description of the $\mathfrak{g}$-action. We replace $G$ and $\mathfrak{g}$ by $H$ and $\mathfrak{h}$ to match our later notation, and we write $A_{x}$ for the $x$-eigenspace of $A$, where $x$ is a member of the character group $X(H)$. Finally, we use $(-\mid-)$ to denote the Euclidean inner product (or "dot product") in any $k^{r}$.

LEMMA 1.3. - Let $A$ be a $k$-algebra, equipped with a rational action of a torus $H=\left(k^{\times}\right)^{r}$ (by $k$-algebra automorphisms). Identify $\mathfrak{h}=$ Lie $H$ with $k^{r}$, and let $\mathfrak{h}$ act on $A$ by the differential of the $H$-action. Further, identify $\mathbb{Z}^{r}$ with $X(H)$ via the natural pairing

$$
\begin{aligned}
\mathbb{Z}^{r} \times\left(k^{\times}\right)^{r} & \longrightarrow k^{\times} \\
\left(m_{1}, \ldots, m_{r}, h_{1}, \ldots, h_{r}\right) & \longmapsto h_{1}^{m_{1}} h_{2}^{m_{2}} \cdots h_{r}^{m_{r}} .
\end{aligned}
$$

Then $\eta . a=(\eta \mid x) a$ for $\eta \in \mathfrak{h}, a \in A_{x}$, and $x \in X(H)$.

In particular, it follows that the $\mathfrak{h}$-action on $A$ commutes with the $H$-action.

Readers who do not wish to delve into the full theory of actions of algebraic groups may take the formula in Lemma 1.3 as the definition of the $\mathfrak{h}$-action on A.

Whenever we have a torus $H$ acting rationally on a $k$-algebra $A$, we will assume that its Lie algebra $\mathfrak{h}$ correspondingly acts on $A$ by the differential of the $H$-action. We label the action of $H$ on $A$ a rational Poisson action in case $A$ is a Poisson algebra and $H$ acts rationally on $A$ by Poisson automorphisms.

Lemma 1.4. - Let $A$ be a Poisson algebra, equipped with a rational Poisson action of a torus $H$. Then $\mathfrak{h}=\operatorname{Lie} H$ acts on $A$ by Poisson derivations.

Proof. - Let $\eta \in \mathfrak{h}$, and let $a \in A_{x}$ and $b \in A_{y}$ for some $x, y \in X(H)$. Since $h .\{a, b\}=\{h . a, h . b\}=x(h) y(h)\{a, b\}$ for all $h \in H$, we have $\{a, b\} \in A_{x+y}$. Taking account of the identifications in Lemma 1.5, we see that

$$
\eta \cdot\{a, b\}=(\eta \mid x+y)\{a, b\}=((\eta \mid x)+(\eta \mid y))\{a, b\}=\{\eta . a, b\}+\{a, \eta . b\} .
$$

Therefore, since $A$ is $X(H)$-graded, we conclude that $\eta$.(-) is a Poisson derivation on $A$.

TheOREM 1.5. - Let $A=k\left[x_{1}\right]\left[x_{2} ; \alpha_{2}, \delta_{2}\right]_{p} \cdots\left[x_{n} ; \alpha_{n}, \delta_{n}\right]_{p}$ be an iterated Poisson polynomial algebra, supporting a rational action by a torus $H$ such that $x_{1}, \ldots, x_{n}$ are $H$-eigenvectors. Assume that there exist $\eta_{1}, \ldots, \eta_{n} \in \mathfrak{h}=$ Lie $H$ such that $\eta_{i} . x_{j}=\alpha_{i}\left(x_{j}\right)$ for $i>j$ and the $\eta_{i}$-eigenvalue of $x_{i}$ is nonzero for each $i$. Then $A$ has at most $2^{n} H$-stable Poisson primes. 
Remark 1.6. - Here the elements of $H$ are only assumed to act on $A$ by $k$ algebra automorphisms, not necessarily by Poisson automorphisms. However, the assumption of a Poisson action is needed in Corollary 1.7.

Proof. - Set $A_{i}=k\left[x_{1}\right]\left[x_{2} ; \alpha_{2}, \delta_{2}\right]_{p} \cdots\left[x_{i} ; \alpha_{i}, \delta_{i}\right]_{p}$ for $i=0,1, \ldots, n$. In view of $\S 1.2$, the $H$-stable Poisson primes in $A_{i}$ coincide with the $\mathfrak{h}$-stable Poisson primes. Obviously $A_{0}=k$ has only one $\mathfrak{h}$-stable Poisson prime. Now let $i<n$ and assume that $A_{i}$ has a finite number, say $n_{i}$, of $\mathfrak{h}$-stable Poisson primes. It follows from the relations $\eta_{i} . x_{j}=\alpha_{i}\left(x_{j}\right)$ that the action of $\eta_{i}$ on $A_{i}$ coincides with $\alpha_{i}$. Proposition 1.2 now implies that the number of $\mathfrak{h}$-stable Poisson primes in $A_{i+1}$ is at most $2 n_{i}$. The theorem follows.

Corollary 1.7. - Let $A=k\left[x_{1}\right]\left[x_{2} ; \alpha_{2}, \delta_{2}\right]_{p} \cdots\left[x_{n} ; \alpha_{n}, \delta_{n}\right]_{p}$ be an iterated Poisson polynomial algebra, supporting a rational Poisson action by a torus $H$ such that $x_{1}, \ldots, x_{n}$ are $H$-eigenvectors. Assume that there exist $\eta_{1}, \ldots, \eta_{n} \in$ $\mathfrak{h}=$ Lie $H$ such that $\eta_{i} . x_{j}=\alpha_{i}\left(x_{j}\right)$ for $i>j$ and the $\eta_{i}$-eigenvalue of $x_{i}$ is nonzero for each $i$. Then A satisfies the Poisson Dixmier-Moeglin equivalence.

Proof. - Theorem 1.5 and [8, Theorem 4.3].

Theorem 1.5 and Corollary 1.7 can be extended to certain non-polynomial affine Poisson algebras as follows.

Proposition 1.8. - Let $A$ be a Poisson algebra which is generated (as an algebra) by a Poisson subalgebra $B$ together with a single element $x$. Assume that $A$ supports a rational Poisson action by a torus $H$ such that $B$ is $H$-stable and $x$ is an $H$-eigenvector. Moreover, assume that there is some $\eta_{0} \in \mathfrak{h}=\operatorname{Lie} H$ such that $\{x, b\}-\left(\eta_{0} . b\right) x \in B$ for all $b \in B$, and such that the $\eta_{0}$-eigenvalue of $x$ is nonzero. Then there are at most twice as many $H$-stable Poisson primes in $A$ as in $B$.

Proof. - We show that $A$ is an epimorphic image of a Poisson polynomial ring $\widehat{A}=B[X ; \alpha, \delta]_{p}$ to which Proposition 1.2 applies. Let $\alpha$ denote the restriction of $\eta_{0} .(-)$ to $B$. Then, by Lemma 1.4, $\alpha$ is a Poisson derivation on $B$, and, by hypothesis, $\delta(b):=\{x, b\}-\alpha(b) x \in B$ for all $b \in B$. Since $\{x,-\}$ and $\alpha$ are derivations, so is $\delta$. For $b, b^{\prime} \in B$, we compute that

$$
\begin{aligned}
\delta\left(\left\{b, b^{\prime}\right\}\right)= & \left\{x,\left\{b, b^{\prime}\right\}\right\}-\alpha\left(\left\{b, b^{\prime}\right\}\right) x \\
= & -\left\{b,\left\{b^{\prime}, x\right\}\right\}-\left\{b^{\prime},\{x, b\}\right\}-\left(\left\{\alpha(b), b^{\prime}\right\}+\left\{b, \alpha\left(b^{\prime}\right)\right\}\right) x \\
= & \left(\left\{\{x, b\}, b^{\prime}\right\}-\left\{\alpha(b) x, b^{\prime}\right\}\right)+\left\{\alpha(b) x, b^{\prime}\right\}-\left\{\alpha(b), b^{\prime}\right\} x \\
& \quad+\left(\left\{b,\left\{x, b^{\prime}\right\}\right\}-\left\{b, \alpha\left(b^{\prime}\right) x\right\}\right)+\left\{b, \alpha\left(b^{\prime}\right) x\right\}-\left\{b, \alpha\left(b^{\prime}\right)\right\} x
\end{aligned}
$$




$$
\begin{aligned}
& =\left\{\delta(b), b^{\prime}\right\}+\left\{b, \delta\left(b^{\prime}\right)\right\}+\alpha(b)\left\{x, b^{\prime}\right\}+\alpha\left(b^{\prime}\right)\{b, x\} \\
& =\left\{\delta(b), b^{\prime}\right\}+\left\{b, \delta\left(b^{\prime}\right)\right\}+\alpha(b)\left(\alpha\left(b^{\prime}\right) x+\delta\left(b^{\prime}\right)\right)+\alpha\left(b^{\prime}\right)(-\alpha(b) x-\delta(b)) \\
& =\left\{\delta(b), b^{\prime}\right\}+\left\{b, \delta\left(b^{\prime}\right)\right\}+\alpha(b) \delta\left(b^{\prime}\right)-\delta(b) \alpha\left(b^{\prime}\right) .
\end{aligned}
$$

Thus, the conditions for the existence of the Poisson polynomial ring $\widehat{A}=$ $B[X ; \alpha, \delta]_{p}$ are verified.

Let $f \in X(H)$ be the $H$-eigenvalue of $x$. The action of $H$ on $B$ extends to a rational action of $H$ on $\widehat{A}$ (by algebra automorphisms, at least) such that $X$ is an $H$-eigenvector with $H$-eigenvalue $f$. (It is easily checked that $H$ acts on $\widehat{A}$ by Poisson automorphisms, but we shall not need this fact.) Since $x$ and $X$ have the same $H$-eigenvalue, they have the same $\mathfrak{h}$-eigenvalue, and hence the same $\eta_{0}$-eigenvalue. Thus, the $\eta_{0}$-eigenvalue of $X$ is nonzero. Since $\eta_{0}$ acts as a derivation on $\widehat{A}$ extending $\alpha$, Proposition 1.2 now implies that for each Poisson prime $Q$ of $B$, there are at most two $\eta_{0}$-stable Poisson primes of $\widehat{A}$ that contract to $Q$. All $H$-stable ideals of $\widehat{A}$ are $\mathfrak{h}$-stable and thus $\eta_{0}$-stable, and so we conclude that for each $H$-stable Poisson prime $Q$ of $B$, there are at most two $H$-stable Poisson primes of $\widehat{A}$ that contract to $Q$. Thus, there are at most twice as many $H$-stable Poisson primes in $\widehat{A}$ as in $B$.

Finally, the identity map on $B$ extends to a $k$-algebra surjection $\pi: \widehat{A} \rightarrow A$ such that $\pi(X)=x$. Obviously $\pi$ preserves brackets of elements of $B$, and

$$
\pi(\{X, b\})=\alpha(b) x+\delta(b)=\{x, b\}=\{\pi(X), \pi(b)\}
$$

for $b \in B$, from which we see that $\pi$ is a Poisson homomorphism. By construction, $\pi$ is also $H$-equivariant. Hence, the set map $\pi^{-1}$ embeds the collection of $H$-stable Poisson primes of $A$ into the collection of $H$-stable Poisson primes of $\widehat{A}$. Therefore, there are at most twice as many $H$-stable Poisson primes in $A$ as in $B$.

Theorem 1.9. - Let $A$ be a Poisson algebra, equipped with a rational Poisson action by a torus $H$. Assume that $A$ is generated by $H$-eigenvectors $x_{1}, \ldots, x_{n}$, and that there exist $\eta_{1}, \ldots, \eta_{n} \in \mathfrak{h}=$ Lie $H$ such that

1. $\left\{x_{i}, x_{j}\right\}-\left(\eta_{i} . x_{j}\right) x_{i} \in k\left\langle x_{1}, \ldots, x_{i-1}\right\rangle$ for all $i>j$;

2. For all $i$, the $\eta_{i}$-eigenvalue of $x_{i}$ is nonzero.

Then $A$ has at most $2^{n} H$-stable Poisson primes, and A satisfies the Poisson Dixmier-Moeglin equivalence.

Proof. - The first conclusion is clear when $n=0$. Now let $n>0$, and assume that the subalgebra $B:=k\left\langle x_{1}, \ldots, x_{n-1}\right\rangle$ has at most $2^{n-1} H$-stable Poisson primes. Note that the map $\delta_{n}:=\left\{x_{n},-\right\}-\left(\eta_{n} .-\right) x_{n}$ is a derivation on $A$. Since, by hypothesis, $\delta_{n}\left(x_{j}\right) \in B$ for all $j<n$, it follows that $\delta_{n}(B) \subseteq B$. 
Since the $\eta_{n}$-eigenvalue of $x_{n}$ is nonzero, Proposition 1.8 implies that $A$ has at most twice as many $H$-stable Poisson primes as $B$, thus at most $2^{n}$.

The final conclusion now follows from [8, Theorem 4.3].

\section{Poisson polynomial algebras satisfying the Poisson Dixmier-Moeglin equivalence}

We show that semiclassical limits of many standard quantum algebra constructions yield Poisson polynomial algebras to which Theorem 1.5 and Corollary 1.7 apply.

2.1. Semiclassical limits. - Suppose that $R$ is a commutative principal ideal domain, containing $k$, and that $\hbar \in R$ with $\hbar R$ a maximal ideal of $R$. If $B$ is a torsionfree $R$-algebra for which the quotient $A:=B / \hbar B$ is commutative, then there is a well-defined bilinear map $\frac{1}{\hbar}[-,-]: B \times B \rightarrow B$, which induces a Poisson bracket on $A$ (e.g., see [2, §III.5.4]). The Poisson algebra $A$ is known as the semiclassical limit (or quasiclassical limit) of $B$, or of the family of algebras $(B / \mathfrak{m} B)_{\mathfrak{m} \in \max R}$.

There are two standard choices for $R$ in quantum algebra constructions. In single parameter cases, we take $R$ to be a Laurent polynomial ring $k\left[q, q^{-1}\right]$ and $\hbar=q-1$, while multiparameter cases are usually best handled by taking $R$ to be a formal power series algebra $k[[\hbar]]$. In the latter situation, we use the abbreviation

$$
e(\alpha):=\exp (\alpha \hbar)=\sum_{i=0}^{\infty} \frac{1}{i !} \alpha^{i} \hbar^{i}
$$

for $\alpha \in k$. Note that $e(\alpha+\beta)=e(\alpha) e(\beta)$ for $\alpha, \beta \in k$.

2.2. Semiclassical limits of quantum affine spaces. - (a) Suppose that $\mathbf{q}=\left(q_{i j}\right)$ is an $n \times n$ multiplicatively antisymmetric matrix over $k$, that is, $q_{i i}=1$ and $q_{j i}=q_{i j}^{-1}$ for all $i, j$. The corresponding multiparameter quantized coordinate ring of affine $n$-space is the $k$-algebra $\theta_{\mathbf{q}}\left(k^{n}\right)$ with generators $x_{1}, \ldots, x_{n}$ and relations $x_{i} x_{j}=q_{i j} x_{j} x_{i}$ for all $i, j$. Similarly, if $\mathbf{q}$ is a multiplicatively antisymmetric matrix over a commutative ring $R$, we can form the $R$-algebra $\theta_{\mathbf{q}}\left(R^{n}\right)$. Observe that $\theta_{\mathbf{q}}\left(R^{n}\right)$ is an iterated skew polynomial algebra over $R$, and hence a free $R$-module.

(b) To write the semiclassical limits of the above algebras, a change of notation is convenient. Let $\mathbf{q}$ now be an (additively) antisymmetric matrix in $M_{n}(k)$. Since the matrix $e(\mathbf{q})=\left(e\left(q_{i j}\right)\right)$ is a multiplicatively antisymmetric matrix over $k[[\hbar]]$, we can form the $k[[\hbar]]$-algebra $B=\theta_{e(\mathbf{q})}\left(k[[\hbar]]^{n}\right)$. As noted in (a), $B$ is a free $k[[\hbar]]$-module, and hence it is torsionfree over $k[[\hbar]]$. We identify the quotient $A=B / \hbar B$ with the polynomial algebra $k\left[x_{1}, \ldots, x_{n}\right]$. 
Since this algebra is commutative, it inherits a Poisson bracket such that $\left\{x_{i}, x_{j}\right\}=q_{i j} x_{i} x_{j}$ for all $i, j$.

(c) There is a rational action of the torus $H=\left(k^{\times}\right)^{n}$ on $A$ such that

$$
\left(h_{1}, \ldots, h_{n}\right) \cdot x_{i}=h_{i} x_{i}
$$

for $\left(h_{1}, \ldots, h_{n}\right) \in H$ and $i=1, \ldots, n$. This action preserves the Poisson bracket on the indeterminates, that is, $h .\left\{x_{i}, x_{j}\right\}=\left\{h . x_{i}, h . x_{j}\right\}$ for $h \in H$ and all $i, j$. Consequently, it is a Poisson action. In this case, Theorem 1.5 is not needed, since $A$ clearly has exactly $2^{n} H$-stable primes, namely the ideals $\left\langle x_{i} \mid i \in I\right\rangle$ for $I \subseteq\{1, \ldots, n\}$. That $A$ satisfies the Poisson Dixmier-Moeglin equivalence was shown in [8, Example 4.6].

2.3. Semiclassical limits of quantum matrices. - (a) Given a nonzero scalar $\lambda \in$ $k^{\times}$and a multiplicatively antisymmetric matrix $\mathbf{p}=\left(p_{i j}\right) \in M_{n}\left(k^{\times}\right)$, the multiparameter quantum $n \times n$ matrix algebra $\Theta_{\lambda, \mathbf{p}}\left(M_{n}(k)\right)$ is the $k$-algebra with generators $X_{i j}$ for $i, j=1, \ldots, n$ and relations

$$
X_{l m} X_{i j}= \begin{cases}p_{l i} p_{j m} X_{i j} X_{l m}+(\lambda-1) p_{l i} X_{i m} X_{l j} & (l>i, m>j) \\ \lambda p_{l i} p_{j m} X_{i j} X_{l m} & (l>i, m \leq j) \\ p_{j m} X_{i j} X_{l m} & (l=i, m>j) .\end{cases}
$$

The standard single parameter case is recovered when $\lambda=q^{-2}$ and $p_{i j}=q$ for all $i>j$. When $\lambda=1$, we just have a multiparameter quantum affine $n^{2}$-space, $\vartheta_{\mathbf{q}}\left(k^{n^{2}}\right)$, for suitable $\mathbf{q}$.

(b) Now let $\mathbf{p}$ be an antisymmetric matrix in $M_{n}(k)$, and $\lambda \in k$ an arbitrary scalar. Form the algebra $B=\theta_{e(\lambda), e(\mathbf{p})}\left(M_{n}(k[[\hbar]])\right)$, and identify the quotient $A=B / \hbar B$ with the polynomial algebra over $k$ in the indeterminates $X_{i j}$, that is, $A=\theta\left(M_{n}(k)\right)$. One can check directly that $B$ is an iterated skew polynomial algebra over $k[[\hbar]]$. Alternatively, it is known that $\Theta_{e(\lambda), e(\mathbf{p})}\left(M_{n}(k((\hbar)))\right)$ is an iterated skew polynomial algebra over the field $k((\hbar))$, and one observes that the automorphisms and skew derivations of this structure map the relevant $k[[\hbar]]-$ subalgebras into themselves. Either way, we conclude that $B$ is torsionfree over $k[[\hbar]]$.

Now $\Theta\left(M_{n}(k)\right)$ inherits a Poisson bracket such that

$$
\left\{X_{l m}, X_{i j}\right\}= \begin{cases}\left(p_{l i}+p_{j m}\right) X_{i j} X_{l m}+\lambda X_{i m} X_{l j} & (l>i, m>j) \\ \left(\lambda+p_{l i}+p_{j m}\right) X_{i j} X_{l m} & (l>i, m \leq j) \\ p_{j m} X_{i j} X_{l m} & (l=i, m>j) .\end{cases}
$$

When $\lambda=0$, we have a semiclassical limit of a quantum affine $n^{2}$-space, a case covered in $\S 2.2$. Hence, we now assume that $\lambda \neq 0$. 
Observe that

$$
\begin{aligned}
\left\{X_{l m}, k\left[X_{i j} \mid(i, j)\right.\right. & \left.\left.<_{\operatorname{lex}}(l, m)\right]\right\} \subseteq \\
& k\left[X_{i j} \mid(i, j)<_{\operatorname{lex}}(l, m)\right] X_{l m}+k\left[X_{i j} \mid(i, j)<_{\operatorname{lex}}(l, m)\right]
\end{aligned}
$$

for all $l, m$, and so when the $X_{i j}$ are adjoined in lexicographic order, $\Theta\left(M_{n}(k)\right)$ is an iterated Poisson polynomial algebra of the form

$$
\vartheta\left(M_{n}(k)\right)=k\left[X_{11}\right]\left[X_{12} ; \alpha_{12}, \delta_{12}\right]_{p} \cdots\left[X_{n n} ; \alpha_{n n}, \delta_{n n}\right]_{p} .
$$

In view of $(7)$, we have

$$
\alpha_{l m}\left(X_{i j}\right)= \begin{cases}\left(p_{l i}+p_{j m}\right) X_{i j} & (l>i, m>j) \\ \left(\lambda+p_{l i}+p_{j m}\right) X_{i j} & (l>i, m \leq j) \\ p_{j m} X_{i j} & (l=i, m>j) .\end{cases}
$$

(c) There is a rational action of the torus $H=\left(k^{\times}\right)^{2 n}$ on $\Theta\left(M_{n}(k)\right)$ such that

$$
h . X_{i j}=h_{i} h_{n+j} X_{i j} \quad\left(h=\left(h_{1}, \ldots, h_{2 n}\right) \in H\right)
$$

for all $i, j$, and it is clear from (7) that this is a Poisson action. If we identify $\mathbb{Z}^{2 n}$ with $X(H)$ as in Lemma 1.3 , then each $X_{i j}$ has $H$-eigenvalue $\epsilon_{i}+\epsilon_{n+j}$, where $\left(\epsilon_{1}, \ldots, \epsilon_{2 n}\right)$ is the canonical basis for $\mathbb{Z}^{2 n}$. Hence, the differential of the $H$-action gives an action of $\mathfrak{h}=$ Lie $H=k^{2 n}$ on $\Theta\left(M_{n}(k)\right)$ by derivations such that

$$
\eta \cdot X_{i j}=\left(\eta_{i}+\eta_{n+j}\right) X_{i j} \quad\left(\eta=\left(\eta_{1}, \ldots, \eta_{2 n}\right) \in \mathfrak{h}\right)
$$

for all $i, j$.

(d) For $l, m=1, \ldots, n$, define $\eta_{l m} \in \mathfrak{h}$ as follows:

$$
\eta_{l m}=\left(p_{l 1}, \ldots, p_{l n}, p_{1 m}, \ldots, p_{m-1, m}, \lambda, \lambda+p_{m+1, m}, \ldots, \lambda+p_{n m}\right) .
$$

Then observe that $\eta_{l m} . X_{i j}=\alpha_{l m}\left(X_{i j}\right)$ for $(i, j)<_{\text {lex }}(l, m)$, and that the $\eta_{l m^{-}}$ eigenvalue of $X_{l m}$ is $\lambda$. Since we have assumed that $\lambda \neq 0$, the hypotheses of Theorem 1.5 and Corollary 1.7 are satisfied. Therefore $A$ has at most $2^{n^{2}} H$ stable Poisson primes, and $A$ satisfies the Poisson Dixmier-Moeglin equivalence. The case of the latter result with $n=2$ and the standard Poisson bracket was established by $\mathrm{Oh}$ in [27, Proposition 2.3].

\subsection{Semiclassical limits of quantum symplectic and even-dimensional euclidean} spaces. - Multiparameter versions of the mentioned quantum algebras are instances of the algebras $K_{n, \Gamma}^{P, Q}(k)$ introduced by Horton [15], and we treat that general class.

(a) Let $\Gamma=\left(\gamma_{i j}\right) \in M_{n}\left(k^{\times}\right)$be a multiplicatively antisymmetric matrix, and let $P=\left(p_{1}, \ldots p_{n}\right)$ and $Q=\left(q_{1}, \ldots q_{n}\right)$ be vectors in $\left(k^{\times}\right)^{n}$ such that $p_{i} \neq q_{i}$ 
for all $i$. Then $K_{n, \Gamma}^{P, Q}(k)$ is the $k$-algebra with generators $x_{1}, y_{1}, \ldots, x_{n}, y_{n}$ and relations

$$
\begin{aligned}
y_{i} y_{j} & =\gamma_{i j} y_{j} y_{i} & & (\text { all } i, j) \\
x_{i} y_{j} & =p_{j} \gamma_{j i} y_{j} x_{i} & & (i<j) \\
x_{i} y_{j} & =q_{j} \gamma_{j i} y_{j} x_{i} & & (i>j) \\
x_{i} x_{j} & =q_{i} p_{j}^{-1} \gamma_{i j} x_{j} x_{i} & & (i<j) \\
x_{i} y_{i} & =q_{i} y_{i} x_{i}+\sum_{\ell<i}\left(q_{\ell}-p_{\ell}\right) y_{\ell} x_{\ell} & & (\text { all } i) .
\end{aligned}
$$

See [15, Examples 1.3-1.7] for the choices of parameters which yield the standard quantum symplectic and even-dimensional euclidean spaces, and related algebras. This construction can be performed over a commutative ring $R$, assuming the $p_{i}, q_{i}$, and $\gamma_{i j}$ are units in $R$, and as in [15, Proposition 2.5], $K_{n, \Gamma}^{P, Q}(R)$ is an iterated skew polynomial algebra over $R$.

(b) Now let $\Gamma$ be an antisymmetric matrix in $M_{n}(k)$, and let $P$ and $Q$ be vectors in $k^{n}$, with $p_{i} \neq q_{i}$ for all $i$. Form the algebra $B=$ $K_{n, e(\Gamma)}^{e(P), e(Q)}(k[[\hbar]])$, and identify the quotient $A=B / \hbar B$ with the polynomial algebra $k\left[x_{1}, y_{1}, \ldots, x_{n}, y_{n}\right]$. Now $B$ is an iterated skew polynomial algebra over $k[[\hbar]]$, so it is a torsionfree $k[[\hbar]]$-module. Hence, $A$ inherits a Poisson bracket such that

$$
\begin{aligned}
\left\{y_{i}, y_{j}\right\} & =\gamma_{i j} y_{i} y_{j} & & (\text { all } i, j) \\
\left\{x_{i}, y_{j}\right\} & =\left(p_{j}+\gamma_{j i}\right) x_{i} y_{j} & & (i<j) \\
\left\{x_{i}, y_{j}\right\} & =\left(q_{j}+\gamma_{j i}\right) x_{i} y_{j} & & (i>j) \\
\left\{x_{i}, x_{j}\right\} & =\left(q_{i}-p_{j}+\gamma_{i j}\right) x_{i} x_{j} & & (i<j) \\
\left\{x_{i}, y_{i}\right\} & =q_{i} x_{i} y_{i}+\sum_{\ell<i}\left(q_{\ell}-p_{\ell}\right) x_{\ell} y_{\ell} & & (\text { all } i) .
\end{aligned}
$$

This Poisson algebra $A$ was introduced by Oh in [29] and denoted $A_{n, \Gamma}^{P, Q}(k)$. It is an iterated Poisson polynomial algebra of the form

$$
A=k\left[x_{1}\right]\left[y_{1} ; \alpha_{1}, \delta_{1}\right]_{p}\left[x_{2} ; \alpha_{2}^{\prime}\right]_{p}\left[y_{2} ; \alpha_{2}, \delta_{2}\right]_{p} \cdots\left[x_{n} ; \alpha_{n}^{\prime}\right]_{p}\left[y_{n} ; \alpha_{n}, \delta_{n}\right]_{p},
$$

such that

$$
\begin{array}{ll}
\alpha_{j}\left(x_{i}\right)=\left(-p_{j}+\gamma_{i j}\right) x_{i} & \alpha_{j}\left(y_{i}\right)=\gamma_{j i} y_{i} \\
\alpha_{j}\left(x_{j}\right)=-q_{j} x_{j} & \\
\alpha_{j}^{\prime}\left(x_{i}\right)=\left(-q_{i}+p_{j}+\gamma_{j i}\right) x_{i} & \alpha_{j}^{\prime}\left(y_{i}\right)=\left(q_{i}+\gamma_{i j}\right) y_{i}
\end{array}
$$

for all $i<j$. 
(c) There is a rational Poisson action of the torus $H=\left(k^{\times}\right)^{n+1}$ on $A$ such that

$$
h . x_{i}=h_{i} x_{i} \quad h . y_{i}=h_{1} h_{n+1} h_{i}^{-1} y_{i}
$$

for $h \in H$. Then $\mathfrak{h}=$ Lie $H=k^{n+1}$ acts on $A$ by derivations such that

$$
\eta \cdot x_{i}=\eta_{i} x_{i} \quad \eta \cdot y_{i}=\left(\eta_{1}+\eta_{n+1}-\eta_{i}\right) y_{i}
$$

for $\eta \in \mathfrak{h}$.

(d) Define $\eta_{j}, \eta_{j}^{\prime} \in \mathfrak{h}$ as follows:

$$
\begin{array}{ll}
\eta_{1}=\left(-q_{1}, 0, \ldots, 0,1\right) & \\
\eta_{j}=\left(-p_{j}+\gamma_{1 j}, \ldots,-p_{j}+\gamma_{j-1, j},-q_{j}, 0, \ldots, 0, \gamma_{j 1}\right) & (j>1) \\
\eta_{j}^{\prime}=\left(-q_{1}+p_{j}+\gamma_{j 1}, \ldots,-q_{n}+p_{j}+\gamma_{j n}, q_{1}+\gamma_{1 j}\right) & (j>1) .
\end{array}
$$

Note first that $\eta_{1} . x_{1}=\alpha_{1}\left(x_{1}\right)$, and that the $\eta_{1}$-eigenvalue of $y_{1}$ is 1 . For $j>1$, we have $\eta_{j} . x_{i}=\alpha_{j}\left(x_{i}\right)$ for $i \leq j$ and $\eta_{j} . y_{i}=\alpha_{j}\left(y_{i}\right)$ for $i<j$, and the $\eta_{j}$ eigenvalue of $y_{j}$ is $q_{j}-p_{j}$. Finally, we have $\eta_{j}^{\prime} \cdot x_{i}=\alpha_{j}^{\prime}\left(x_{i}\right)$ and $\eta_{j}^{\prime} \cdot y_{i}=\alpha_{j}^{\prime}\left(y_{i}\right)$ for $i<j$, and the $\eta_{j}^{\prime}$-eigenvalue of $x_{j}$ is $p_{j}-q_{j}$. Thus, the hypotheses of Theorem 1.5 and Corollary 1.7 are satisfied. We conclude that $A$ has at most $2^{2 n} H$-stable Poisson primes, and that it satisfies the Poisson Dixmier-Moeglin equivalence. The case $n=2$ of the latter result was established by $\mathrm{Oh}$ in [28, Theorem 3.5].

2.5. Semiclassical limits of quantum odd-dimensional euclidean spaces. - Multiparameter versions of quantum euclidean spaces in the odd-dimensional case can be constructed analogously to the even-dimensional case treated in [15]. Since these algebras have not (to our knowledge) appeared in the literature, we take the opportunity to introduce them here.

(a) As for the $2 n$-dimensional case, let $\Gamma=\left(\gamma_{i j}\right) \in M_{n}\left(k^{\times}\right)$be a multiplicatively antisymmetric matrix, and let $P=\left(p_{1}, \ldots p_{n}\right)$ and $Q=\left(q_{1}, \ldots q_{n}\right)$ be vectors in $\left(k^{\times}\right)^{n}$ such that $p_{i} \neq q_{i}$ for all $i$. Further, let $\lambda \in k$, and assume that each $p_{i}$ has a square root in $k^{\times}$, which we fix and label $p_{i}^{1 / 2}$. Define $K_{n, \Gamma}^{P, Q, \lambda}(k)$ 
to be the $k$-algebra with generators $z_{0}, x_{1}, y_{1}, \ldots, x_{n}, y_{n}$ and relations

$$
\begin{aligned}
z_{0} x_{i} & =p_{i}^{-1 / 2} x_{i} z_{0} \\
z_{0} y_{i} & =p_{i}^{1 / 2} y_{i} z_{0} \\
y_{i} y_{j} & =\gamma_{i j} y_{j} y_{i} \\
x_{i} y_{j} & =p_{j} \gamma_{j i} y_{j} x_{i} \\
x_{i} y_{j} & =q_{j} \gamma_{j i} y_{j} x_{i} \\
x_{i} x_{j} & =q_{i} p_{j}^{-1} \gamma_{i j} x_{j} x_{i} \\
x_{i} y_{i} & =q_{i} y_{i} x_{i}+\sum_{\ell<i}\left(q_{\ell}-p_{\ell}\right) y_{\ell} x_{\ell}+\lambda z_{0}^{2}
\end{aligned}
$$

The standard single parameter algebra corresponds to the case where the $p_{i}=$ $q^{-2}$, the $q_{i}=1$, the $\gamma_{i j}=q^{-1}$ for $i<j$, and $\lambda=(q-1) q^{n-(1 / 2)}$. (This requires a change of variables, as in [23, §§ 2.1, 2.2], [26, Example 5], or [15, Example 1.5].) On the other hand, if we take $\lambda=1$ and all the $p_{i}=1$ (with $p_{i}^{1 / 2}=1$ ), then $z_{0}$ is central in $K_{n, \Gamma}^{P, Q, \lambda}(k)$, and $K_{n, \Gamma}^{P, Q, \lambda}(k) /\left\langle z_{0}-1\right\rangle$ is the multiparameter quantized Weyl algebra $A_{n}^{Q, \Gamma}(k)$ (see, e.g., [2, §I.2.6]).

This construction can be performed over a commutative ring $R$, assuming the relevant parameters are units in $R$ and the $p_{i}$ have square roots in $R$. As in the even-dimensional case [15, Proposition 2.5], $K_{n, \Gamma}^{P, Q, \lambda}(R)$ is an iterated skew polynomial algebra over $R$.

(b) Now let $\Gamma$ be an antisymmetric matrix in $M_{n}(k)$, let $P$ and $Q$ be vectors in $k^{n}$ with $p_{i} \neq q_{i}$ for all $i$, and let $\lambda \in k$. Form the algebra $B=$ $K_{n, e(\Gamma)}^{e(P), e(Q), \lambda \hbar}(k[[\hbar]])$, and identify the quotient $A=B / \hbar B$ with the polynomial algebra $k\left[z_{0}, x_{1}, y_{1}, \ldots, x_{n}, y_{n}\right]$. Here we have used $\lambda \hbar$ rather than $e(\lambda)$ to ensure commutativity of $B / \hbar B$, and we take $e\left(p_{i} / 2\right)$ as the chosen square root of $e\left(p_{i}\right)$. Since $B$ is an iterated skew polynomial algebra over $k[[\hbar]]$, it is a torsionfree $k[[\hbar]]$-module. Hence, $A$ inherits a Poisson bracket such that

$$
\begin{aligned}
& \left\{z_{0}, x_{i}\right\}=-\left(p_{i} / 2\right) z_{0} x_{i} \\
& \left\{z_{0}, y_{i}\right\}=\left(p_{i} / 2\right) z_{0} y_{i} \\
& \left\{y_{i}, y_{j}\right\}=\gamma_{i j} y_{i} y_{j} \\
& \left\{x_{i}, y_{j}\right\}=\left(p_{j}+\gamma_{j i}\right) x_{i} y_{j} \\
& \left\{x_{i}, y_{j}\right\}=\left(q_{j}+\gamma_{j i}\right) x_{i} y_{j} \\
& \left\{x_{i}, x_{j}\right\}=\left(q_{i}-p_{j}+\gamma_{i j}\right) x_{i} x_{j} \\
& \left\{x_{i}, y_{i}\right\}=q_{i} x_{i} y_{i}+\sum_{\ell<i}\left(q_{\ell}-p_{\ell}\right) x_{\ell} y_{\ell}+\lambda z_{0}^{2}
\end{aligned}
$$


This Poisson algebra is an iterated Poisson polynomial algebra of the form

$$
A=k\left[z_{0}\right]\left[x_{1} ; \alpha_{1}^{\prime}\right]_{p}\left[y_{1} ; \alpha_{1}, \delta_{1}\right]_{p}\left[x_{2} ; \alpha_{2}^{\prime}\right]_{p}\left[y_{2} ; \alpha_{2}, \delta_{2}\right]_{p} \cdots\left[x_{n} ; \alpha_{n}^{\prime}\right]_{p}\left[y_{n} ; \alpha_{n}, \delta_{n}\right]_{p} .
$$

(c) There is a rational Poisson action of the torus $H=\left(k^{\times}\right)^{n+1}$ on $A$ such that

$$
\begin{array}{ll}
h . x_{i}=h_{i} x_{i} & h . z_{0}=h_{n+1} z_{0} \\
\text { h. } y_{i}=h_{n+1}^{2} h_{i}^{-1} y_{i} &
\end{array}
$$

for $h \in H$. We leave it to the reader to check that the hypotheses of Theorem 1.5 and Corollary 1.7 are satisfied. We conclude that $A$ has at most $2^{2 n+1} H$-stable Poisson primes, and that it satisfies the Poisson Dixmier-Moeglin equivalence.

2.6. Semiclassical limits of quantum symmetric matrices. - Fix a positive integer $n$.

(a) Coordinate rings of quantum symmetric $n \times n$ matrices have been introduced by Noumi [25, Theorem 4.3, Proposition 4.4, and comments following the proof] and Kamita [19, Theorem 0.2]. As in [11, §5.5], we take the case of Noumi's algebra with all parameters equal to 1, which agrees with Kamita's algebra after interchanging the scalar parameter $q$ with $q^{-1}$. This is a $k$-algebra with generators $y_{i j}$ for $1 \leq i \leq j \leq n$. If the construction is instead performed over a rational function field $k(q)$, the $k\left[q^{ \pm 1}\right]$-subalgebra $B$ generated by the $y_{i j}$ is then an iterated skew polynomial algebra over $k\left[q^{ \pm 1}\right]$, and the quotient $A=B /(q-1) B$ can be identified with the polynomial ring $k\left[y_{i j} \mid 1 \leq i \leq j \leq n\right]$. Hence, $A$ inherits a Poisson bracket, which has the following form, as calculated in $[11, \S 5.5]$ :

$\left\{y_{i j}, y_{l m}\right\}=(\operatorname{sign}(l-j)+\operatorname{sign}(m-i)) y_{i l} y_{j m}+(\operatorname{sign}(l-i)+\operatorname{sign}(m-j)) y_{i m} y_{j l}$

for $i \leq j$ and $l \leq m$, where $\operatorname{sign}(t)$ is 1,0 , or -1 according as $t$ is positive, zero, or negative, and where $y_{t s}=y_{s t}$ if needed. The Poisson algebra $A$ is an iterated Poisson polynomial algebra of the form

$$
A=k\left[y_{11}\right]\left[y_{12} ; \alpha_{12}, \delta_{12}\right]_{p} \cdots\left[y_{n n} ; \alpha_{n n}, \delta_{n n}\right]_{p},
$$

where the indeterminates $y_{i j}$ (for $1 \leq i \leq j \leq n$ ) have been adjoined in lexicographic order, and where

$\alpha_{l m}\left(y_{i j}\right)= \begin{cases}-y_{i j} & ((i=l<j<m) \text { or }(i<l<j=m) \text { or }(i<j=l<m)) \\ -2 y_{i j} & ((i=j=l<m) \text { or }(i<j=l=m)) \\ 0 & \text { (otherwise) }\end{cases}$

for $l \leq m$ and $i \leq j$ with $(i, j)<_{\operatorname{lex}}(l, m)$ 
(b) There is a rational Poisson action of the torus $H=\left(k^{\times}\right)^{n}$ on $A$ such that $h . y_{i j}=h_{i} h_{j} y_{i j}$ for all $h \in H$ and all $i, j$. Then $\mathfrak{h}=\operatorname{Lie} H=k^{n}$ acts on $A$ by derivations such that $\eta \cdot y_{i j}=\left(\eta_{i}+\eta_{j}\right) y_{i j}$ for all $\eta \in \mathfrak{h}$ and all $i$, $j$. Let $\left(\epsilon_{1}, \ldots, \epsilon_{n}\right)$ denote the canonical basis for $\mathfrak{h}$, and set $\eta_{l m}=-\epsilon_{l}-\epsilon_{m}$ for $1 \leq l \leq m \leq n$. Then $\eta_{l m} . y_{i j}=\alpha_{l m}\left(y_{i j}\right)$ for $(i, j)<_{\operatorname{lex}}(l, m)$, and the $\eta_{l m}$-eigenvalue of $y_{l m}$ is either -2 or -4 (depending on whether $l<m$ or $l=m$ ). Thus, the hypotheses of Theorem 1.5 and Corollary 1.7 are satisfied. We conclude that $A$ has at most $2^{n(n+1) / 2} H$-stable Poisson primes, and that it satisfies the Poisson Dixmier-Moeglin equivalence.

2.7. Semiclassical limits of quantum antisymmetric matrices. - Fix a positive integer $n$.

(a) The coordinate ring of quantum antisymmetric $n \times n$ matrices was introduced by Strickland in [32, Section 1]; it is a $k$-algebra with generators $y_{i j}$ for $1 \leq i<j \leq n$ and relations involving a scalar $q \in k^{\times}$. If the construction is instead performed over $k(q)$, the $k\left[q^{ \pm 1}\right]$-subalgebra $B$ generated by the $y_{i j}$ is then an iterated skew polynomial algebra over $k\left[q^{ \pm 1}\right]$, and the quotient $A=B /(q-1) B$ can be identified with the polynomial ring $k\left[y_{i j} \mid 1 \leq i<j \leq n\right]$. Hence, $A$ inherits a Poisson bracket, which has the following form, as noted in [11, §5.6(b)]. (The factor 2 in [11] does not appear here, because we are using Strickland's construction without changing $q$ to $q^{1 / 2}$.)

$\left\{y_{i j}, y_{l m}\right\}=(\operatorname{sign}(l-j)+\operatorname{sign}(m-i)) y_{i l} y_{j m}-(\operatorname{sign}(l-i)+\operatorname{sign}(m-j)) y_{i m} y_{j l}$

for $i<j$ and $l<m$, where $y_{t s}=-y_{s t}$ and $y_{s s}=0$ if needed. The Poisson algebra $A$ is an iterated Poisson polynomial algebra of the form

$$
A=k\left[y_{12}\right]\left[y_{13} ; \alpha_{13}, \delta_{13}\right]_{p} \cdots\left[y_{n-1, n} ; \alpha_{n-1, n}, \delta_{n-1, n}\right]_{p},
$$

where the indeterminates $y_{i j}$ (for $1 \leq i<j \leq n$ ) have been adjoined in lexicographic order, and where

$$
\alpha_{l m}\left(y_{i j}\right)= \begin{cases}-y_{i j} & (\text { if }|\{i, j\} \cap\{l, m\}|=1) \\ 0 & \text { (otherwise) }\end{cases}
$$

for $l<m$ and $i<j$ with $(i, j)<_{\text {lex }}(l, m)$.

(b) There is a rational Poisson action of the torus $H=\left(k^{\times}\right)^{n}$ on $A$ such that $h . y_{i j}=h_{i} h_{j} y_{i j}$ for all $h \in H$ and all $i, j$. Then $\mathfrak{h}=\operatorname{Lie} H=k^{n}$ acts on $A$ by derivations such that $\eta \cdot y_{i j}=\left(\eta_{i}+\eta_{j}\right) y_{i j}$ for all $\eta \in \mathfrak{h}$ and all $i, j$. Let $\left(\epsilon_{1}, \ldots, \epsilon_{n}\right)$ denote the canonical basis for $\mathfrak{h}$, and set $\eta_{l m}=-\epsilon_{l}-\epsilon_{m}$ for $1 \leq l<m \leq n$. Then $\eta_{l m} . y_{i j}=\alpha_{l m}\left(y_{i j}\right)$ for $(i, j)<_{\operatorname{lex}}(l, m)$, and the $\eta_{l m}$-eigenvalue of $y_{l m}$ is -2 . Thus, the hypotheses of Theorem 1.5 and Corollary 1.7 are satisfied. We 
conclude that $A$ has at most $2^{n(n-1) / 2} H$-stable Poisson primes, and that it satisfies the Poisson Dixmier-Moeglin equivalence.

\section{Fraction fields of Poisson prime quotients}

We now turn to the Poisson structure of fields of fractions of Poisson prime quotients of iterated Poisson polynomial rings.

3.1. - Recall from $\S 0.6$ the notation $k_{\boldsymbol{\lambda}}\left[x_{1}, \ldots, x_{n}\right]$ for the Poisson algebra based on the polynomial ring $k\left[x_{1}, \ldots, x_{n}\right]$ with $\left\{x_{i}, x_{j}\right\}=\lambda_{i j} x_{i} x_{j}$ for all $i, j$, where $\boldsymbol{\lambda}=\left(\lambda_{i j}\right)$ is an antisymmetric matrix in $M_{n}(k)$. The corresponding Poisson Laurent polynomial algebra and Poisson field are denoted $k_{\boldsymbol{\lambda}}\left[x_{1}^{ \pm 1}, \ldots, x_{n}^{ \pm 1}\right]$ and $k_{\boldsymbol{\lambda}}\left(x_{1}, \ldots, x_{n}\right)$, respectively.

The algebra $k_{\boldsymbol{\lambda}}\left[x_{1}^{ \pm 1}, \ldots, x_{n}^{ \pm 1}\right]$ can be identified with the group algebra $k \Gamma$, where $\Gamma=\mathbb{Z}^{n}$, by writing monomials in the $x_{i}$ in the form $x^{\alpha}=x_{1}^{\alpha_{1}} x_{2}^{\alpha_{2}} \cdots x_{n}^{\alpha_{n}}$ for elements $\alpha=\left(\alpha_{1}, \ldots, \alpha_{n}\right) \in \Gamma$. There is then an antisymmetric bilinear form $b: \Gamma \times \Gamma \rightarrow k$ such that

$$
b(\alpha, \beta)=\sum_{i, j=1}^{n} \alpha_{i} \lambda_{i j} \beta_{j}
$$

for $\alpha, \beta \in \Gamma$, and $\left\{x^{\alpha}, x^{\beta}\right\}=b(\alpha, \beta) x^{\alpha+\beta}$ for $\alpha, \beta \in \Gamma$. Conversely, if $b$ is any $k$-valued antisymmetric bilinear form on $\Gamma$, there is a Poisson bracket on $k \Gamma$ such that $\left\{x^{\alpha}, x^{\beta}\right\}=b(\alpha, \beta) x^{\alpha+\beta}$ for $\alpha, \beta \in \Gamma$. We denote this Poisson algebra by $k_{b} \Gamma$. The following facts about $k_{b} \Gamma$ are well known. See, for instance, [35, Lemma 1.2] where they are proved in the case $k=\mathbb{C}$; the arguments are valid over arbitrary base fields.

LEMMA 3.1. - Let $\Gamma=\mathbb{Z}^{n}$ for some $n \in \mathbb{N}$, let $b$ be a $k$-valued antisymmetric bilinear form on $\Gamma$, and let $k_{b} \Gamma$ be the Poisson algebra based on $k \Gamma$ described in $\S 3.1$. Set

$$
\Gamma_{b}=\{\alpha \in \Gamma \mid b(\alpha,-) \equiv 0\},
$$

a subgroup of $\Gamma$. Then $Z_{p}\left(k_{b} \Gamma\right)=k \Gamma_{b}$, and every Poisson ideal of $k_{b} \Gamma$ is generated by its intersection with $Z_{p}\left(k_{b} \Gamma\right)$.

Corollary 3.2. - Let $\Gamma$ and $b$ be as in Lemma 3.1. If $Z_{p}\left(k_{b} \Gamma\right)=k$, then $k_{b} \Gamma$ is Poisson simple, that is, its only Poisson ideals are 0 and $k_{b} \Gamma$.

We can now determine the structure of the fields of fractions of Poisson prime factors of the algebras $k_{\boldsymbol{\lambda}}\left[x_{1}, \ldots, x_{n}\right]$. The method is a Poisson version of the proof of [9, Theorem 2.1]. 
TheOrem 3.3. - Let $A=k_{\boldsymbol{\lambda}}\left[x_{1}, \ldots, x_{n}\right]$ for some antisymmetric matrix $\boldsymbol{\lambda} \in$ $M_{n}(k)$, and let $P$ be a Poisson prime ideal of $A$. Then there exist a field extension $K \supseteq k$ and an antisymmetric matrix $\boldsymbol{\mu} \in M_{m}(k)$, for some $m \leq n$, such that Fract $A / P \cong K_{\boldsymbol{\mu}}\left(y_{1}, \ldots, y_{m}\right)$ (as Poisson algebras).

In fact, $\boldsymbol{\mu}$ is the upper left $m \times m$ submatrix of $\boldsymbol{\sigma} \boldsymbol{\lambda} \boldsymbol{\sigma}^{\text {tr }}$, for some $\boldsymbol{\sigma} \in G L_{n}(\mathbb{Z})$.

Proof. - Write $\boldsymbol{\lambda}=\left(\lambda_{i j}\right)$.

If $I=\left\{i \in\{1, \ldots, n\} \mid x_{i} \notin P\right\}$ and $\boldsymbol{\lambda}^{\prime}$ is the submatrix of $\boldsymbol{\lambda}$ consisting of the rows and columns indexed by $I$, then there is a Poisson prime ideal $P^{\prime}$ in $k_{\boldsymbol{\lambda}^{\prime}}\left[x_{i} \mid i \in I\right]$ such that $A / P \cong k_{\boldsymbol{\lambda}^{\prime}}\left[x_{i} \mid i \in I\right] / P^{\prime}$. Thus, there is no loss of generality in assuming that $x_{i} \notin P$ for all $i$. Now $P$ induces a Poisson prime ideal $Q$ in the algebra $B=k_{\boldsymbol{\lambda}}\left[x_{1}^{ \pm 1}, \ldots, x_{n}^{ \pm 1}\right]$ such that $Q \cap A=P$, and Fract $A / P \cong$ Fract $B / Q$ (as Poisson algebras).

Write $B=k_{b} \Gamma$ as in $\S 3.1$, where $\Gamma=\mathbb{Z}^{n}$ and $b$ is the $k$-valued antisymmetric bilinear form on $\Gamma$ obtained from $\boldsymbol{\lambda}$. Set

$$
\Gamma_{Q}=\left\{\alpha \in \Gamma \mid x^{\alpha}+Q \in Z_{p}(B / Q)\right\},
$$

and observe that $\Gamma_{Q}$ is a subgroup of $\Gamma$. We claim that $\Gamma / \Gamma_{Q}$ is torsionfree. If $\alpha \in \Gamma$ and $t \alpha \in \Gamma_{Q}$ for some $t \in \mathbb{N}$, then $\left(x^{\alpha}+Q\right)^{t}=x^{t \alpha}+Q$ lies in $Z_{p}(B / Q)$, whence

$$
t\left(x^{\alpha}+Q\right)^{t-1}\left\{x^{\alpha}+Q, B / Q\right\}=\left\{\left(x^{\alpha}+Q\right)^{t}, B / Q\right\}=0 .
$$

Since $t\left(x^{\alpha}+Q\right)^{t-1}$ is a unit in $B / Q$, it follows that $\left\{x^{\alpha}+Q, B / Q\right\}=0$, that is, $\alpha \in \Gamma_{Q}$. Therefore $\Gamma / \Gamma_{Q}$ is torsionfree, as claimed.

Now $\Gamma / \Gamma_{Q}$ is a free abelian group of rank $m \leq n$, so there exists a basis $\left(\epsilon_{1}, \ldots, \epsilon_{n}\right)$ for $\Gamma$ such that $\Gamma_{Q}$ is generated by $\left\{\epsilon_{m+1}, \ldots, \epsilon_{n}\right\}$. Let $\left(\gamma_{1}, \ldots, \gamma_{n}\right)$ denote the standard basis for $\Gamma$. There is a matrix $\boldsymbol{\sigma}=\left(\sigma_{r s}\right) \in G L_{n}(\mathbb{Z})$ such that each $\epsilon_{r}=\sum_{s=1}^{n} \sigma_{r s} \gamma_{s}$, and we set $\boldsymbol{\xi}$ equal to the antisymmetric matrix $\boldsymbol{\sigma} \boldsymbol{\lambda} \boldsymbol{\sigma}^{\text {tr }} \in M_{n}(k)$. Then $B=k_{\boldsymbol{\xi}}\left[z_{1}^{ \pm 1}, \ldots, z_{n}^{ \pm 1}\right]$, where $z_{i}=x^{\epsilon_{i}}$ for $i=1, \ldots, n$. Hence, after replacing the $x_{i}$ and $\boldsymbol{\lambda}$ by the $z_{i}$ and $\boldsymbol{\xi}$, we may assume that $\left(\epsilon_{1}, \ldots, \epsilon_{n}\right)$ is the standard basis for $\Gamma$. In particular, this means that $x_{i}+Q \in$ $Z_{p}(B / Q)$ for $i=m+1, \ldots, n$.

Set $K=$ Fract $Z_{p}(B / Q) \subseteq$ Fract $B / Q$, let $\boldsymbol{\mu} \in M_{m}(k)$ be the (antisymmetric) upper left $m \times m$ submatrix of $\boldsymbol{\lambda}$, and form the Poisson $K$-algebra $C=$ $K_{\boldsymbol{\mu}}\left[y_{1}^{ \pm 1}, \ldots, y_{m}^{ \pm 1}\right]$. There is a $K$-algebra homomorphism $\phi: C \rightarrow$ Fract $B / Q$ such that $\phi\left(y_{i}\right)=x_{i}+Q$ for $i=1, \ldots, m$, and $\phi$ is a Poisson homomorphism because

$$
\left\{\phi\left(y_{i}\right), \phi\left(y_{j}\right)\right\}=\left\{x_{i}, x_{j}\right\}+Q=\lambda_{i j} x_{i} x_{j}+Q=\phi\left(\left\{y_{i}, y_{j}\right\}\right)
$$

for $i, j=1, \ldots, m$. Since $x_{i}+Q \in K$ for $i>m$, the image of $\phi$ contains all the $x_{i}+Q$, and so

$$
\text { Fract } \phi(C)=\text { Fract } B / Q \cong \text { Fract } A / P \text {. }
$$


Thus, it only remains to show that $\phi$ is injective.

We claim that $C$ is Poisson simple. Identify $C$ with $K_{c} \Delta$ in the notation of $\S 3.1$, where $\Delta=\mathbb{Z}^{m}$ and $c$ is the $k$-valued antisymmetric bilinear form on $\Delta$ obtained from $\boldsymbol{\mu}$. Further, identify $\Delta$ with the subgroup of $\Gamma$ generated by $\epsilon_{1}, \ldots, \epsilon_{m}$; then $\Gamma=\Delta \oplus \Gamma_{Q}$ and $c$ is the restriction of $b$ to $\Delta \times \Delta$. We use Lemma 3.1 to prove that $Z_{p}(C)=K$, after which Corollary 3.2 will imply that $C$ is Poisson simple. Thus, let $\alpha \in \Delta_{c}$, that is, $\alpha \in \Delta$ and $c(\alpha,-) \equiv 0$. For $j=1, \ldots, m$, we obtain

$$
0=c\left(\alpha, \epsilon_{j}\right)=\sum_{i=1}^{m} \alpha_{i} \lambda_{i j},
$$

and consequently

$$
\left\{x^{\alpha}, x_{j}\right\}=\sum_{i=1}^{m} \alpha_{i} \lambda_{i j} x^{\alpha} x_{j}=0 .
$$

Since $\left\{x^{\alpha}, x_{j}\right\} \in Q$ for $j>m$ (because $x_{j}+Q \in Z_{p}(B / Q)$ ), it follows that $x^{\alpha}+Q \in Z_{p}(B / Q)$, and so $\alpha \in \Gamma_{Q}$. However, $\Delta \cap \Gamma_{Q}=0$, forcing $\alpha=0$. We have proved that $\Delta_{c}=0$, and hence $Z_{p}(C)=K$ by Lemma 3.1. Corollary 3.2 now implies that $C$ is Poisson simple, as claimed.

Since $\operatorname{ker} \phi$ is a Poisson ideal of $C$, it must be zero. Therefore $\phi$ is injective, and the proof is complete.

We next construct a Poisson version of the derivation-deleting map introduced by Cauchon in [4, Section 2].

Lemma 3.4. - Let $A=B[x ; \alpha, \delta]_{p}$ be a Poisson polynomial algebra. Assume that $\delta$ is locally nilpotent, and that $\alpha \delta=\delta(\alpha+s)$ for some $s \in k^{\times}$. Then the rule

$$
\theta(b)=\sum_{n=0}^{\infty} \frac{1}{n !}\left(\frac{-1}{s}\right)^{n} \delta^{n}(b) x^{-n}
$$

defines a k-algebra homomorphism $\theta: B \rightarrow B\left[x^{ \pm 1}\right]$, and

$$
\{x, \theta(b)\}=\theta \alpha(b) x
$$

for all $b \in B$.

Proof. - Note first that $(21)$ at least defines a $k$-linear map $\theta: B \rightarrow B\left[x^{ \pm 1}\right]$, and that $\theta(1)=1$. We compute that

$$
\begin{aligned}
\theta(a b) & =\sum_{n=0}^{\infty} \frac{1}{n !}\left(\frac{-1}{s}\right)^{n} \delta^{n}(a b) x^{-n}=\sum_{n=0}^{\infty} \frac{1}{n !}\left(\frac{-1}{s}\right)^{n} \sum_{l=0}^{n}\left(\begin{array}{l}
n \\
l
\end{array}\right) \delta^{l}(a) \delta^{n-l}(b) x^{-n} \\
& =\sum_{l, m=0}^{\infty} \frac{1}{l !} \frac{1}{m !}\left(\frac{-1}{s}\right)^{l+m} \delta^{l}(a) \delta^{m}(b) x^{-l-m}=\theta(a) \theta(b)
\end{aligned}
$$


for all $a, b \in A$. Therefore $\theta$ is a $k$-algebra homomorphism.

For $b \in B$, we have

$$
\begin{aligned}
\{x, \theta(b)\} & =\sum_{n=0}^{\infty} \frac{1}{n !}\left(\frac{-1}{s}\right)^{n}\left(\alpha \delta^{n}(b) x+\delta^{n+1}(b)\right) x^{-n} \\
& =\theta \delta(b)+\sum_{n=0}^{\infty} \frac{1}{n !}\left(\frac{-1}{s}\right)^{n} \delta^{n}(\alpha+n s)(b) x^{1-n} \\
& =\theta \delta(b)+\theta \alpha(b) x-\sum_{n=1}^{\infty} \frac{1}{(n-1) !}\left(\frac{-1}{s}\right)^{n-1} \delta^{n}(b) x^{1-n} \\
& =\theta \delta(b)+\theta \alpha(b) x-\theta \delta(b)=\theta \alpha(b) x .
\end{aligned}
$$

This proves (22).

Lemma 3.5. - Let $A=B[x ; \alpha, \delta]_{p}$ be a Poisson polynomial algebra, and assume that $\alpha \delta=\delta(\alpha+s)$ for some $s \in k$. Then

(23) $\delta^{n}(\{a, b\})=\sum_{l+m=n}\left(\begin{array}{l}n \\ l\end{array}\right)\left(\left\{\delta^{l}(a), \delta^{m}(b)\right\}+m \delta^{l} \alpha(a) \delta^{m}(b)-l \delta^{l}(a) \delta^{m} \alpha(b)\right)$

for all $a, b \in B$ and $n \geq 0$.

Proof. - Let $L$ denote the $k[x]$-linear map $\{x,-\}: A \rightarrow A$. Because of the Jacobi identity for the Poisson bracket, $L$ is a Poisson derivation on $A$. Hence, $L$ satisfies the Leibniz Rule

$$
L^{n}(\{a, b\})=\sum_{l+m=n}\left(\begin{array}{l}
n \\
l
\end{array}\right)\left\{L^{l}(a), L^{m}(b)\right\}
$$

for $a, b \in A$ and $n \geq 0$. Next, write $\equiv_{1}$ and $\equiv_{2}$ for congruence modulo the ideals $(x)$ and $\left(x^{2}\right)$ in $A$, respectively. We claim that

$$
L^{n}(a) \equiv_{2} \delta^{n}(a)+\left[n \delta^{n-1} \alpha(a)+\frac{n(n-1) s}{2} \delta^{n-1}(a)\right] x
$$

for $a \in B$ and $n \geq 0$. This holds trivially when $n=0$, and by construction when $n=1$.

If (25) holds for some $a \in B$ and some $n$, then

$$
\begin{aligned}
L^{n+1}(a) & \equiv{ }_{2} \delta^{n+1}(a)+\alpha \delta^{n}(a) x+\left[n \delta^{n} \alpha(a)+\frac{n(n-1) s}{2} \delta^{n}(a)\right] x \\
& \equiv{ }_{2} \delta^{n+1}(a)+\left[(n+1) \delta^{n} \alpha(a)+\frac{n(n+1) s}{2} \delta^{n}(a)\right] x,
\end{aligned}
$$

because $\alpha \delta^{n}=\delta^{n}(\alpha+n s)$. Thus, by induction, (25) holds. 
Now let $a, b \in B$ and $n \geq 0$. In view of (24) and (25), we have (26)

$$
\begin{aligned}
& L^{n}(\{a, b\}) \equiv \sum_{l+m=n}\left(\begin{array}{l}
n \\
l
\end{array}\right)\left\{\delta^{l}(a)+\left[l \delta^{l-1} \alpha(a)+\frac{l(l-1) s}{2} \delta^{l-1}(a)\right] x,\right. \\
&\left.\delta^{m}(b)+\left[m \delta^{m-1} \alpha(b)+\frac{m(m-1) s}{2} \delta^{m-1}(b)\right] x\right\} \\
& \equiv \sum_{1} \sum_{l+m=n}\left(\begin{array}{l}
n \\
l
\end{array}\right)\left(\left\{\delta^{l}(a), \delta^{m}(b)\right\}+U_{l m}+V_{l m}\right),
\end{aligned}
$$

where

$$
\begin{aligned}
& U_{l m}=l \delta^{l-1} \alpha(a) \delta^{m+1}(b)-m \delta^{m-1} \alpha(b) \delta^{l+1}(a) \\
& V_{l m}=\frac{l(l-1) s}{2} \delta^{l-1}(a) \delta^{m+1}(b)-\frac{m(m-1) s}{2} \delta^{m-1}(b) \delta^{l+1}(a) .
\end{aligned}
$$

Observe that

$$
\begin{aligned}
\sum_{l+m=n}\left(\begin{array}{c}
n \\
l
\end{array}\right) U_{l m}= & \sum_{\substack{l+m=n \\
m>0}}\left(\begin{array}{c}
n \\
l+1
\end{array}\right)(l+1) \delta^{l} \alpha(a) \delta^{m}(b) \\
& -\sum_{\substack{l+m=n \\
l>0}}\left(\begin{array}{c}
n \\
l-1
\end{array}\right)(m+1) \delta^{l}(a) \delta^{m} \alpha(b) \\
= & \sum_{l+m=n}\left(\begin{array}{c}
n \\
l
\end{array}\right)\left(m \delta^{l} \alpha(a) \delta^{m}(b)-l \delta^{l}(a) \delta^{m} \alpha(b)\right)
\end{aligned}
$$

while

$$
\begin{aligned}
\sum_{l+m=n}\left(\begin{array}{c}
n \\
l
\end{array}\right) V_{l m}= & \frac{s}{2} \sum_{\substack{l+m=n \\
m>0}}\left(\begin{array}{c}
n \\
l+1
\end{array}\right)(l+1) l \delta^{l}(a) \delta^{m}(b) \\
& \quad-\frac{s}{2} \sum_{\substack{l+m=n \\
l>0}}\left(\begin{array}{c}
n \\
l-1
\end{array}\right)(m+1) m \delta^{l}(a) \delta^{m}(b) \\
= & 0 .
\end{aligned}
$$

Combining (26), (27), and (28), we obtain

$$
L^{n}(\{a, b\}) \equiv_{1} \sum_{l+m=n}\left(\begin{array}{l}
n \\
l
\end{array}\right)\left(\left\{\delta^{l}(a), \delta^{m}(b)\right\}+m \delta^{l} \alpha(a) \delta^{m}(b)-l \delta^{l}(a) \delta^{m} \alpha(b)\right) .
$$

Since $L^{n}(\{a, b\}) \equiv_{1} \delta^{n}(\{a, b\}),(29)$ implies (23). 
Lemma 3.6. - Under the hypotheses of Lemma 3.4, the map $\theta$ is a Poisson homomorphism from $B$ to $B\left[x^{ \pm 1} ; \alpha, \delta\right]_{p}$.

Proof. - For $a, b \in B$, we compute, using (22), that

$$
\begin{aligned}
\{\theta(a), \theta(b)\} & =\sum_{l=0}^{\infty} \frac{1}{l !}\left(\frac{-1}{s}\right)^{l}\left\{\delta^{l}(a) x^{-l}, \theta(b)\right\} \\
& =\sum_{l=0}^{\infty} \frac{1}{l !}\left(\frac{-1}{s}\right)^{l}\left(\left\{\delta^{l}(a), \theta(b)\right\} x^{-l}-l \delta^{l}(a)\{x, \theta(b)\} x^{-l-1}\right) \\
& =\sum_{l=0}^{\infty} \frac{1}{l !}\left(\frac{-1}{s}\right)^{l}\left(\left\{\delta^{l}(a), \theta(b)\right\}-l \delta^{l}(a) \theta \alpha(b)\right) x^{-l} \\
& =\sum_{l, m=0}^{\infty} \frac{1}{l ! m !}\left(\frac{-1}{s}\right)^{l+m}\left(\left\{\delta^{l}(a), \delta^{m}(b) x^{-m}\right\}-l \delta^{l}(a) \delta^{m} \alpha(b) x^{-m}\right) x^{-l} \\
& =\sum_{l, m=0}^{\infty} \frac{1}{l ! m !}\left(\frac{-1}{s}\right)^{l+m}\left(C_{l m}+D_{l m}\right) x^{-l-m},
\end{aligned}
$$

where

$$
\begin{aligned}
& C_{l m}=\left\{\delta^{l}(a), \delta^{m}(b)\right\}+m \delta^{l} \alpha(a) \delta^{m}(b)-l \delta^{l}(a) \delta^{m} \alpha(b) \\
& D_{l m}=l m s \delta^{l}(a) \delta^{m}(b)+m \delta^{l+1}(a) \delta^{m}(b) x^{-1}
\end{aligned}
$$

for all $l, m$. The contribution of the $D_{l m}$ terms to the sum in (30) is

$$
\begin{aligned}
\sum_{l, m=0}^{\infty} \frac{1}{l ! m !}\left(\frac{-1}{s}\right)^{l+m} & {\left[l m s \delta^{l}(a) \delta^{m}(b) x^{-l-m}+m \delta^{l+1}(a) \delta^{m}(b) x^{-l-m-1}\right] } \\
= & \sum_{l, m=1}^{\infty} \frac{1}{(l-1) !(m-1) !} \frac{(-1)^{l+m}}{s^{l+m-1}} \delta^{l}(a) \delta^{m}(b) x^{-l-m} \\
& +\sum_{t, m=1}^{\infty} \frac{1}{(t-1) !(m-1) !}\left(\frac{-1}{s}\right)^{t-1+m} \delta^{t}(a) \delta^{m}(b) x^{-t-m} \\
= & 0 .
\end{aligned}
$$

Because of (31), (32), and Lemma 3.5, we may simplify (30) to

$$
\begin{aligned}
\{\theta(a), \theta(b)\} & =\sum_{l, m=0}^{\infty} \frac{1}{l ! m !}\left(\frac{-1}{s}\right)^{l+m} C_{l m} x^{-l-m} \\
& =\sum_{n=0}^{\infty} \frac{1}{n !}\left(\frac{-1}{s}\right)^{n} \delta^{n}(\{a, b\}) x^{-n}=\theta(\{a, b\}) .
\end{aligned}
$$


Therefore $\theta$ preserves the Poisson bracket.

THEOREM 3.7. - Under the hypotheses of Lemma 3.4, the map $\theta$ extends uniquely to an isomorphism of Poisson Laurent polynomial algebras,

$$
\theta: B\left[y^{ \pm 1} ; \alpha\right]_{p} \stackrel{\cong}{\longrightarrow} B\left[x^{ \pm 1} ; \alpha, \delta\right]_{p},
$$

such that $\theta(y)=x$.

Proof. - First, $\theta$ extends uniquely to a $k$-algebra homomorphism $B\left[y^{ \pm 1}\right] \rightarrow$ $B\left[x^{ \pm 1}\right]$ such that $\theta(y)=x$. In view of $(22)$ and Lemma 3.6, the forms $\theta(\{-,-\})$ and $\{\theta(-), \theta(-)\}$ agree on pairs of elements from $B \cup\left\{y^{ \pm 1}\right\}$, from which we see that they agree on pairs of elements from $A$. Hence, the extended map $\theta$ is a Poisson homomorphism, and it only remains to show that $\theta$ is bijective.

For surjectivity, we already have $x^{ \pm 1}=\theta\left(y^{ \pm 1}\right)$, and so we just need to see that $B$ is contained in the image of $\theta$. Given $b \in B$, there is some $l \geq 0$ such that $\delta^{l}(b)=0$, and we proceed by induction on $l$. If $l \leq 1$, then $\delta(b)=0$ and $\theta(b)=b$. Now let $l>1$, and write $\theta(b)=b+\sum_{n=1}^{l-1} \lambda_{n} \delta^{n}(b) x^{-n}$ for some $\lambda_{n} \in k$. Since $\delta^{l-1}\left(\delta^{n}(b)\right)=0$ for $n=1, \ldots, l-1$, we can assume by induction that $\delta^{1}(b), \ldots, \delta^{l-1}(b)$ are in the image of $\theta$. Consequently, $\theta(b)-b$ is in the image of $\theta$, and thus $b$ is in the image of $\theta$. This establishes the induction step, and proves that $\theta$ is surjective.

Let $p \in B\left[y^{ \pm 1}\right]$ be nonzero, and write $p=\sum_{i=l}^{m} b_{i} y^{i}$ for some $b_{i} \in B$ and some integers $l \leq m$, with $b_{m} \neq 0$. Each of the terms $\theta\left(b_{i} y^{i}\right)$ is a Laurent polynomial of the form $b_{i} x^{i}+$ [lower terms]. Hence, $\theta(p)=b_{m} x^{m}+$ [lower terms], and thus $\theta(p) \neq 0$. Therefore $\theta$ is injective.

The following is the main result addressing our quadratic version of the Poisson Gelfand-Kirillov problem. It is a Poisson version of [4, Théorème 6.1.1].

ThEOREM 3.8. - Let $A=k\left[x_{1}\right]\left[x_{2} ; \alpha_{2}, \delta_{2}\right]_{p} \cdots\left[x_{n} ; \alpha_{n}, \delta_{n}\right]_{p}$ be an iterated Poisson polynomial algebra such that

(a) $\delta_{i}$ is locally nilpotent for all $i$.

(b) There exist $s_{i} \in k^{\times}$such that $\alpha_{i} \delta_{i}=\delta_{i}\left(\alpha_{i}+s_{i}\right)$ for all $i$.

(c) There exist $\lambda_{i j} \in k$ such that $\alpha_{i}\left(x_{j}\right)=\lambda_{i j} x_{j}$ for all $i>j$.

Let $\boldsymbol{\lambda}=\left(\lambda_{i j}\right)$ be the antisymmetric matrix in $M_{n}(k)$ whose entries below the diagonal agree with the scalars in (c). Then:

(1) Fract $A \cong k_{\boldsymbol{\lambda}}\left(y_{1}, \ldots, y_{n}\right)$.

(2) For any Poisson prime ideal $P$ of $A$, there exist a field extension $K \supseteq$ $k$ and an antisymmetric matrix $\boldsymbol{\mu} \in M_{m}(k)$, for some $m \leq n$, such that Fract $A / P \cong K_{\boldsymbol{\mu}}\left(y_{1}, \ldots, y_{m}\right)$ (as Poisson algebras). In fact, $\boldsymbol{\mu}$ is the upper left $m \times m$ submatrix of $\boldsymbol{\sigma} \boldsymbol{\lambda} \boldsymbol{\sigma}^{\mathrm{tr}}$, for some $\boldsymbol{\sigma} \in G L_{n}(\mathbb{Z})$. 
Proof. - Let $P$ be an arbitrary Poisson prime ideal of $A$, and set $B=$ $k_{\boldsymbol{\lambda}}\left[z_{1}, \ldots, z_{n}\right]$. In view of Theorem 3.3 , it suffices to show that

1. "(*)" Fract $A / P \cong$ Fract $B / Q$ for some Poisson prime ideal $Q$ of $B$, where $Q=0$ if $P=0$.

We prove $\left(^{*}\right)$ via a triple induction: first, with respect to $n$; second, with respect to the number $d$ of indices $i$ for which $\delta_{i} \neq 0$; and third (downward), with respect to the maximum index $t$ for which $\delta_{t} \neq 0$. (If $d=0$, we take $t=n+1$.) Since there is nothing to prove if $n=1$ or $t=n+1$, we may assume that $n \geq 2$ and $t \leq n$.

CAse 1: $x_{n} \in P$. Then there exists a Poisson prime ideal $P^{\prime}$ in the algebra

$$
A^{\prime}=k\left[x_{1}\right]\left[x_{2} ; \alpha_{2}, \delta_{2}\right]_{p} \cdots\left[x_{n-1} ; \alpha_{n-1}, \delta_{n-1}\right]_{p}
$$

such that $A / P \cong A^{\prime} / P^{\prime}$. By our primary induction, there is a Poisson prime ideal $Q^{\prime}$ in the algebra $B^{\prime}=k_{\boldsymbol{\lambda}^{\prime}}\left[z_{1}, \ldots, z_{n-1}\right]$, where $\lambda^{\prime}$ is the upper left $(n-$ $1) \times(n-1)$ submatrix of $\boldsymbol{\lambda}$, such that Fract $A^{\prime} / P^{\prime} \cong$ Fract $B^{\prime} / Q^{\prime}$. Observe that $Q=Q^{\prime}+B z_{n}$ is a Poisson prime ideal of $B$ such that $B^{\prime} / Q^{\prime} \cong B / Q$. Thus, Fract $A / P \cong$ Fract $B / Q$.

CASE 2: $x_{n} \notin P$ and $t=n$. Then $\delta_{n} \neq 0$. Set

$$
A^{\prime}=k\left[x_{1}\right]\left[x_{2} ; \alpha_{2}, \delta_{2}\right]_{p} \cdots\left[x_{n-1} ; \alpha_{n-1}, \delta_{n-1}\right]_{p}\left[y ; \alpha_{n}\right]_{p}
$$

By Theorem 3.7, $A\left[x_{n}^{-1}\right] \cong A^{\prime}\left[y^{-1}\right]$, and so there exists a Poisson prime ideal $P^{\prime}$ in $A^{\prime}$ such that Fract $A / P \cong$ Fract $A^{\prime} / P^{\prime}$, where $P^{\prime}=0$ if $P=0$. The number of nonzero maps among $\delta_{2}, \ldots, \delta_{n-1}$ is $d-1$. Thus, our secondary induction yields $(*)$ in this case.

CASE 3: $t<n$. Then $\delta_{n}=0$. Since $\left\{x_{n}, x_{1}\right\}=\lambda_{n 1} x_{1} x_{n}$, we see that $\left\{x_{n}, k\left[x_{1}\right]\right\} \subseteq k\left[x_{1}\right] x_{n}$, and so $k\left[x_{1}, x_{n}\right]$ is a Poisson polynomial algebra of the form $k\left[x_{1}\right]\left[x_{n} ; \alpha_{n}^{\prime}\right]_{p}$. For $i=2, \ldots, n-1$, we have

$$
\left\{x_{i}, k\left[x_{1}, \ldots, x_{i-1}\right]\right\} \subseteq k\left[x_{1}, \ldots, x_{i-1}\right] x_{i}+k\left[x_{1}, \ldots, x_{i-1}\right]
$$

and $\left\{x_{i}, x_{n}\right\}=-\lambda_{n i} x_{i} x_{n}=\lambda_{i n} x_{n} x_{i}$, from which it follows that

$$
\left\{x_{i}, k\left[x_{1}, \ldots, x_{i-1}, x_{n}\right]\right\} \subseteq k\left[x_{1}, \ldots, x_{i-1}, x_{n}\right] x_{i}+k\left[x_{1}, \ldots, x_{i-1}, x_{n}\right] .
$$

Hence, we may rewrite $A$ in the form

$$
A=k\left[x_{1}\right]\left[x_{n} ; \alpha_{n}^{\prime}\right]_{p}\left[x_{2} ; \alpha_{2}^{\prime}, \delta_{2}^{\prime}\right]_{p} \cdots\left[x_{n-1} ; \alpha_{n-1}^{\prime}, \delta_{n-1}^{\prime}\right]_{p}
$$

for suitable $\alpha_{i}^{\prime}$ and $\delta_{i}^{\prime}$, such that $\alpha_{i}^{\prime}\left(x_{j}\right)=\lambda_{i j} x_{j}$ for $j<i$ and for $j=n$. Note that $\alpha_{i}^{\prime}$ and $\delta_{i}^{\prime}$ restrict to $\alpha_{i}$ and $\delta_{i}$ on $k\left[x_{1}, \ldots, x_{i-1}\right]$, and that $\delta_{i}^{\prime}\left(x_{n}\right)=0$. It follows easily that $\delta_{i}^{\prime}$ is locally nilpotent, and that $\alpha_{i}^{\prime} \delta_{i}^{\prime}=\delta_{i}^{\prime}\left(\alpha_{i}^{\prime}+s_{i}\right)$. Finally, the map $\delta_{t}^{\prime}$ is nonzero because it restricts to $\delta_{t}$, and it occurs in position $t+1$ in the list $0,0, \delta_{2}^{\prime}, \ldots, \delta_{n-1}^{\prime}$. Thus, our tertiary induction yields $\left(^{*}\right)$ in this case.

Therefore $\left(^{*}\right)$ holds in all cases, and the theorem is proved. 


\section{Poisson polynomial algebras satisfying the quadratic Gelfand-Kirillov property}

We apply Theorem 3.8 to the algebras discussed in Section 2, to obtain the following result.

Theorem 4.1. - Let $A$ be any of the Poisson algebras of $\S \S 2.2(b)$, 2.3(b), 2.4(b), 2.5(b), 2.6(a), 2.7(a). For any Poisson prime ideal $P$ of $A$, there exist a field extension $K \supseteq k$ and an antisymmetric matrix $\boldsymbol{\mu} \in M_{m}(k)$, for some $m$, such that Fract $A / P \cong K_{\boldsymbol{\mu}}\left(y_{1}, \ldots, y_{m}\right)$ (as Poisson algebras). In case $P=0$, we have $K=k$ and $m=\operatorname{tr} \cdot \operatorname{deg}_{k} A$.

In each case, bounds on $m$ and restrictions on $\boldsymbol{\mu}$ can be obtained via Theorem 3.8. We leave details to the interested reader. The form of the Poisson field Fract $A$ is given below.

The examples in Section 2 are already expressed as iterated Poisson polynomial algebras, and so what remains is to establish hypotheses (a), (b), (c) of Theorem 3.8 in each case. For (a), the following observation is helpful: To check that a derivation $\delta$ on an algebra $A$ is locally nilpotent, it suffices to check that $\delta$ is locally nilpotent on a set of algebra generators for $A$. (This follows directly from the Leibniz Rule for $\delta$.) Hypothesis (b) is built into the situation of Corollary 1.7, as follows.

Lemma 4.2. - Let $A=k\left[x_{1}\right]\left[x_{2} ; \alpha_{2}, \delta_{2}\right]_{p} \cdots\left[x_{n} ; \alpha_{n}, \delta_{n}\right]_{p}$ be an iterated Poisson polynomial algebra, supporting a rational Poisson action by a torus $H$ such that $x_{1}, \ldots, x_{n}$ are $H$-eigenvectors. Assume that there exist $\eta_{1}, \ldots, \eta_{n} \in \mathfrak{h}=$ Lie $H$ such that $\eta_{i} . x_{j}=\alpha_{i}\left(x_{j}\right)$ for $i>j$ and the $\eta_{i}$-eigenvalue of $x_{i}$, call it $s_{i}$, is nonzero for each $i$. Then $\alpha_{i} \delta_{i}=\delta_{i}\left(\alpha_{i}+s_{i}\right)$ for all $i$.

Proof. - Fix $i \in\{2, \ldots, n\}$. Since the derivations $\eta_{i} \cdot(-)$ and $\alpha_{i}$ agree on $x_{1}, \ldots, x_{i-1}$, they must agree on the algebra $A_{i-1}=k\left[x_{1}, \ldots, x_{i-1}\right]$. Let $y_{i}$ denote the $H$-eigenvalue of $x_{i}$, so that $x_{i} \in A_{y_{i}}$. Then $s_{i} x_{i}=\eta_{i} \cdot x_{i}=\left(\eta_{i} \mid y_{i}\right) x_{i}$, and so $\left(\eta_{i} \mid y_{i}\right)=s_{i}$.

Consider an $H$-eigenvector $f \in A_{i-1}$, say $f \in A_{z}$ for some $z \in X(H)$, and note that

$$
\left\{x_{i}, f\right\}=\alpha_{i}(f) x_{i}+\delta_{i}(f)=\left(\eta_{i} . f\right) x_{i}+\delta_{i}(f)=\left(\eta_{i} \mid z\right) f x_{i}+\delta_{i}(f) .
$$

As shown in the proof of Lemma $1.4,\left\{x_{i}, f\right\} \in A_{y_{i}+z}$. Since also $f x_{i} \in A_{y_{i}+z}$, we see that $\delta_{i}(f) \in A_{y_{i}+z}$. Consequently,

$$
\begin{aligned}
\alpha_{i} \delta_{i}(f) & =\eta_{i} \cdot \delta_{i}(f)=\left(\eta_{i} \mid y_{i}+z\right) \delta_{i}(f)=\delta_{i}\left(\left(\eta_{i} \mid z\right) f+\left(\eta_{i} \mid y_{i}\right) f\right) \\
& =\delta_{i}\left(\eta_{i} . f+s_{i} f\right)=\delta_{i}\left(\alpha_{i}+s_{i}\right)(f) .
\end{aligned}
$$

The lemma then follows from the rationality of the action of the torus $H$. 
Since we have shown that the examples in Section 2 satisfy the hypotheses of Corollary 1.7, we conclude from Lemma 4.2 that they also satisfy hypothesis (b) of Theorem 3.8.

4.1. - The algebra $A$ of $\S 2.2(\mathrm{~b})$ is just $k_{\mathbf{q}}\left[x_{1}, \ldots, x_{n}\right]$, and Theorem 3.3 applies.

4.2. - Let $A=\Theta\left(M_{n}(k)\right)$ with the Poisson bracket given in (7). As the case $\lambda=0$ is covered by $\S 4$.1, we assume that $\lambda \neq 0$. Condition (c) of Theorem 3.8 is given by (8). The maps $\delta_{l m}$ in this algebra satisfy

$$
\delta_{l m}\left(X_{i j}\right)= \begin{cases}\lambda X_{i m} X_{l j} & (l>i, m>j) \\ 0 & \text { (otherwise) } .\end{cases}
$$

In particular, $\delta_{l m}^{2}\left(X_{i j}\right)=0$ for all $(i, j)<_{\text {lex }}(l, m)$, whence $\delta_{l m}$ is locally nilpotent. Thus, the hypotheses of Theorem 3.8 are satisfied. In particular, the theorem implies that Fract $A \cong k\left(Y_{i j} \mid i, j=1, \ldots, n\right)$ with

$$
\left\{Y_{l m}, Y_{i j}\right\}= \begin{cases}\left(p_{l i}+p_{j m}\right) Y_{i j} Y_{l m} & (l \geq i, m>j) \\ \left(\lambda+p_{l i}+p_{j m}\right) Y_{i j} Y_{l m} & (l>i, m \leq j) .\end{cases}
$$

4.3. - Let $A=A_{n, \Gamma}^{P, Q}(k)$ as in $\S 2.4(\mathrm{~b})$. Condition (c) of Theorem 3.8 is given by (11). The maps $\delta_{i}$ here satisfy

$$
\begin{aligned}
& \delta_{i}\left(x_{j}\right)=\delta_{i}\left(y_{j}\right)=0 \quad(j<i) \\
& \delta_{i}\left(x_{i}\right)=-\sum_{l<i}\left(q_{l}-p_{l}\right) x_{l} y_{l} .
\end{aligned}
$$

Thus $\delta_{i}^{2}$ vanishes on $x_{1}, y_{1}, \ldots, x_{i-1}, y_{i-1}, x_{i}$, whence $\delta_{i}$ is locally nilpotent. In this case, Theorem 3.8 shows that Fract $A \cong k\left(v_{1}, w_{1}, \ldots, v_{n}, w_{n}\right)$ with

$$
\begin{aligned}
\left\{w_{i}, w_{j}\right\} & =\gamma_{i j} w_{i} w_{j} & & (\text { all } i, j) \\
\left\{v_{i}, w_{j}\right\} & =\left(p_{j}+\gamma_{j i}\right) v_{i} w_{j} & & (i<j) \\
\left\{v_{i}, w_{j}\right\} & =\left(q_{j}+\gamma_{j i}\right) v_{i} w_{j} & & (i \geq j) \\
\left\{v_{i}, v_{j}\right\} & =\left(q_{i}-p_{j}+\gamma_{i j}\right) v_{i} v_{j} & & (i<j) .
\end{aligned}
$$

4.4. - Let $A$ be as in $\S 2.5(\mathrm{~b})$. Condition (c) of Theorem 3.8 is clear from (16). The maps $\delta_{i}$ here satisfy

$$
\begin{aligned}
& \delta_{i}\left(x_{j}\right)=\delta_{i}\left(y_{j}\right)=\delta_{i}\left(z_{0}\right)=0 \quad(j<i) \\
& \delta_{i}\left(x_{i}\right)=-\sum_{l<i}\left(q_{l}-p_{l}\right) x_{l} y_{l}-\lambda z_{0}^{2} .
\end{aligned}
$$


Thus $\delta_{i}^{2}$ vanishes on $z_{0}, x_{1}, y_{1}, \ldots, x_{i-1}, y_{i-1}, x_{i}$, whence $\delta_{i}$ is locally nilpotent. We see from Theorem 3.8 that Fract $A \cong k\left(u_{0}, v_{1}, w_{1}, \ldots, v_{n}, w_{n}\right)$ with

$$
\begin{aligned}
\left\{u_{0}, v_{i}\right\} & =-\left(p_{i} / 2\right) u_{0} v_{i} & & \text { (all } i) \\
\left\{u_{0}, w_{i}\right\} & =\left(p_{i} / 2\right) u_{0} w_{i} & & \text { (all } i) \\
\left\{w_{i}, w_{j}\right\} & =\gamma_{i j} w_{i} w_{j} & & (\text { all } i, j) \\
\left\{v_{i}, w_{j}\right\} & =\left(p_{j}+\gamma_{j i}\right) v_{i} w_{j} & & (i<j) \\
\left\{v_{i}, w_{j}\right\} & =\left(q_{j}+\gamma_{j i}\right) v_{i} w_{j} & & (i \geq j) \\
\left\{v_{i}, v_{j}\right\} & =\left(q_{i}-p_{j}+\gamma_{i j}\right) v_{i} v_{j} & & (i<j) .
\end{aligned}
$$

4.5. - Let $A$ be as in $\S 2.6($ a). Condition (c) of Theorem 3.8 is given by (18). The maps $\delta_{l m}$ in this algebra satisfy

$$
\delta_{l m}\left(y_{i j}\right)= \begin{cases}-2 y_{i m} y_{l j} & (i<l \leq j<m) \\ -2 y_{i l} y_{j m}-2 y_{i m} y_{j l} & (i \leq j<l \leq m) \\ 0 & \text { (otherwise) }\end{cases}
$$

for $l \leq m$ and $i \leq j$ with $(i, j)<_{\text {lex }}(l, m)$. It follows that $\delta_{l m}^{3}\left(y_{i j}\right)=0$ for all $(i, j)<_{\operatorname{lex}}(l, m)$, whence $\delta_{l m}$ is locally nilpotent. In this case, Theorem 3.8 implies that Fract $A \cong k\left(z_{i j} \mid 1 \leq i \leq j \leq n\right)$ with

$$
\left\{z_{i j}, z_{l m}\right\}= \begin{cases}z_{i j} z_{l m} & ((i=l<j<m) \text { or }(i<l<j=m) \text { or }(i<j=l<m)) \\ 2 z_{i j} z_{l m} & ((i=j=l<m) \text { or }(i<j=l=m)) \\ 0 & \text { (otherwise) }\end{cases}
$$

for $l \leq m$ and $i \leq j$ with $(i, j)<_{\operatorname{lex}}(l, m)$.

4.6. - Let $A$ be as in $\S 2.7($ a). Condition (c) of Theorem 3.8 is given by (20). The maps $\delta_{l m}$ in this algebra satisfy

$$
\delta_{l m}\left(y_{i j}\right)= \begin{cases}-2 y_{i m} y_{l j} & (i<l<j<m) \\ -2 y_{i l} y_{j m}+2 y_{i m} y_{j l} & (i<j<l<m) \\ 0 & \text { (otherwise) }\end{cases}
$$

for $l<m$ and $i<j$ with $(i, j)<_{\text {lex }}(l, m)$. It follows that $\delta_{l m}^{2}\left(y_{i j}\right)=0$ for all $(i, j)<_{\operatorname{lex}}(l, m)$, whence $\delta_{l m}$ is locally nilpotent. In this case, finally, we see from Theorem 3.8 that Fract $A \cong k\left(z_{i j} \mid 1 \leq i<j \leq n\right)$ with

$$
\left\{z_{i j}, z_{l m}\right\}= \begin{cases}z_{i j} z_{l m} & (\text { if }|\{i, j\} \cap\{l, m\}|=1) \\ 0 & \text { (otherwise) }\end{cases}
$$

for $l<m$ and $i<j$ with $(i, j)<_{\text {lex }}(l, m)$. 


\section{Isomorphism invariants of quadratic Poisson fields}

In this final section of the paper, we address the question of when Poisson fields $k_{\boldsymbol{\lambda}}\left(x_{1}, \ldots, x_{n}\right)$ and $k_{\boldsymbol{\mu}}\left(x_{1}, \ldots, x_{n}\right)$ can be isomorphic. It is easily seen that a sufficient condition is the existence of an invertible integer matrix $A$ such that $\boldsymbol{\mu}=A \boldsymbol{\lambda} A^{\text {tr }}$ (Lemma 5.1), and we show that in a number of cases, this condition is also necessary. The method is to show that the set of matrices $B \boldsymbol{\lambda} B^{\operatorname{tr}}$, for $B \in M_{n}(\mathbb{Z})$, is an invariant of $k_{\boldsymbol{\lambda}}\left(x_{1}, \ldots, x_{n}\right)$. By similar means, we also show that $k_{\boldsymbol{\lambda}}\left(x_{1}, \ldots, x_{n}\right)$ cannot be isomorphic to any Poisson-Weyl field. The invariants we use are Poisson analogs of some invariants introduced by Alev and Dumas in [1].

For purposes of computation in $k_{\boldsymbol{\lambda}}\left(x_{1}, \ldots, x_{n}\right)$, observe that the Poisson bracket of any monomials $x^{a}$ and $x^{b}$ is given by

$$
\left\{x^{a}, x^{b}\right\}_{\boldsymbol{\lambda}}=\sum_{l, m=1}^{n} a_{l} b_{m} \lambda_{l m} x^{a+b}=\left(a \boldsymbol{\lambda} b^{\operatorname{tr}}\right) x^{a+b},
$$

where $a, b \in \mathbb{Z}^{n}$ are viewed as row vectors.

LEMMA 5.1. - Let $\boldsymbol{\lambda}, \boldsymbol{\mu} \in M_{n}(k)$ be antisymmetric, and assume there exists $A \in G L_{n}(\mathbb{Z})$ such that $\boldsymbol{\mu}=A \boldsymbol{\lambda} A^{\text {tr }}$. Then $k_{\boldsymbol{\lambda}}\left(x_{1}, \ldots, x_{n}\right) \cong k_{\boldsymbol{\mu}}\left(x_{1}, \ldots, x_{n}\right)($ as Poisson algebras over $k$ ).

Proof. - Let $a_{1}, \ldots, a_{n}$ denote the rows of $A$, set $y_{i}=x^{a_{i}}$ for $i=1, \ldots, n$, and observe using (33) that

$$
\left\{y_{i}, y_{j}\right\}_{\boldsymbol{\lambda}}=\left(a_{i} \boldsymbol{\lambda} a_{j}^{\mathrm{tr}}\right) y_{i} y_{j}=\boldsymbol{\mu}_{i j} y_{i} y_{j}
$$

for all $i, j$. Since $A$ is invertible, $x_{1}, \ldots, x_{n}$ all lie in $k\left(y_{1}, \ldots, y_{n}\right)$, so the $y_{i}$ are algebraically independent over $k$ and $k\left(y_{1}, \ldots, y_{n}\right)=k\left(x_{1}, \ldots, x_{n}\right)$. Hence, there is a $k$-algebra automorphism $\phi$ of $k\left(x_{1}, \ldots, x_{n}\right)$ sending $y_{i} \mapsto x_{i}$ for all $i$. Since the Poisson brackets $\{-,-\}_{\boldsymbol{\lambda}}$ and $\{-,-\}_{\boldsymbol{\mu}}$ are determined by the values $\left\{y_{i}, y_{j}\right\}_{\boldsymbol{\lambda}}$ and $\left\{x_{i}, x_{j}\right\}_{\boldsymbol{\mu}}$, we conclude that $\phi$ is a Poisson isomorphism of $k_{\boldsymbol{\lambda}}\left(x_{1}, \ldots, x_{n}\right)$ onto $k_{\boldsymbol{\mu}}\left(x_{1}, \ldots, x_{n}\right)$.

Proposition 5.2. - Let $K=k_{\boldsymbol{\lambda}}\left(x_{1}, \ldots, x_{n}\right)$ for some antisymmetric $\boldsymbol{\lambda} \in$ $M_{n}(k)$.

(a) If $B_{\boldsymbol{\lambda}}$ is the $k$-subspace of $K$ spanned by $\{\{f, g\} \mid f, g \in K\}$, then $B_{\boldsymbol{\lambda}} \cap k=$ $\{0\}$.

(b) For any $n$-tuple $y=\left(y_{1}, \ldots, y_{n}\right)$ of nonzero elements of $K$, let $C_{\boldsymbol{\lambda}}(y)$ denote the matrix $\left(\left\{y_{i}, y_{j}\right\}\left(y_{i} y_{j}\right)^{-1}\right) \in M_{n}(K)$. If $C_{\boldsymbol{\lambda}}=\left\{C_{\boldsymbol{\lambda}}(y) \mid y \in\left(K^{\times}\right)^{n}\right\}$, then $C_{\boldsymbol{\lambda}} \cap M_{n}(k)=\left\{A \boldsymbol{\lambda} A^{\operatorname{tr}} \mid A \in M_{n}(\mathbb{Z})\right\}$. 
Proof. - Put the lexicographic order on $\mathbb{Z}^{n}$, and let $L$ denote the corresponding Hahn-Laurent power series field in $x_{1}, \ldots, x_{n}$ (cf. [5, Theorem VII.3.8]; a more detailed treatment can be found in [6, Section 2]). The field $L$ consists of formal series $\sum_{a \in I} \alpha_{a} x^{a}$ where $I$ is a well-ordered subset of $\mathbb{Z}^{n}$ and the $\alpha_{a} \in k$. Finite sums in $L$ are identified with Laurent polynomials in $k\left[x_{1}^{ \pm 1}, \ldots, x_{n}^{ \pm 1}\right]$. Since $L$ is a field, it thus contains (a copy of) $K$. Let $\pi: L \rightarrow k$ be the $k$-linear map that gives the constant term (i.e., the coefficient of $x^{\mathbf{0}}$ ) of elements of $L$. Observe that the Poisson bracket on $K$ extends to $L$ by setting

$$
\{f, g\}=\sum_{i, j=1}^{n} \lambda_{i j} x_{i} x_{j} \frac{\partial f}{\partial x_{i}} \frac{\partial g}{\partial x_{j}}
$$

for $f, g \in L$. This formula gives a well-defined element of $L$ because the supports of $x_{i}\left(\partial f / \partial x_{i}\right)$ and $x_{j}\left(\partial g / \partial x_{j}\right)$ are contained in those of $f$ and $g$.

(a) It suffices to show that $\pi(\{f, g\})=0$ for any $f, g \in L$. Write $f=$ $\sum_{a \in I} \alpha_{a} x^{a}$ and $g=\sum_{b \in J} \beta_{b} x^{b}$ where $I$ and $J$ are well-ordered subsets of $\mathbb{Z}^{n}$ and the $\alpha_{a}, \beta_{b} \in k$. Then

$$
\{f, g\}=\sum_{i, j=1}^{n} \lambda_{i j}\left(\sum_{a \in I} a_{i} \alpha_{a} x^{a}\right)\left(\sum_{b \in J} b_{j} \beta_{b} x^{b}\right)=\sum_{a \in I, b \in J}\left(\sum_{i, j=1}^{n} \lambda_{i j} a_{i} b_{j}\right) \alpha_{a} \beta_{b} x^{a+b},
$$

and consequently

$$
\pi(\{f, g\})=\sum_{\substack{a \in I, b \in J \\ a+b=\mathbf{0}}}\left(\sum_{i, j=1}^{n} \lambda_{i j} a_{i} b_{j}\right) \alpha_{a} \beta_{b}=\sum_{a \in I \cap(-J)}\left(-\sum_{i, j=1}^{n} \lambda_{i j} a_{i} a_{j}\right) \alpha_{a} \beta_{-a} .
$$

Since $\boldsymbol{\lambda}$ is antisymmetric, each of the sums $\sum_{i, j=1}^{n} \lambda_{i j} a_{i} a_{j}$ is zero, and thus $\pi(\{f, g\})=0$, as desired.

(b) It follows from (34) that $A \boldsymbol{\lambda} A^{\text {tr }} \in C_{\boldsymbol{\lambda}}$ for all $A \in M_{n}(\mathbb{Z})$. Hence, it suffices to show that for any $\left(y_{1}, \ldots, y_{n}\right) \in\left(L^{\times}\right)^{n}$, the matrix $\left(\pi\left(\left\{y_{i}, y_{j}\right\}\left(y_{i} y_{j}\right)^{-1}\right)\right)$ has the form $A \boldsymbol{\lambda} A^{\text {tr }}$ for some $A \in M_{n}(\mathbb{Z})$.

Write each $y_{i}=\sum_{a \in I(i)} \alpha_{i a} y^{a}$ where $I(i)$ is a well-ordered subset of $\mathbb{Z}^{n}$ with minimum element $m(i)$, the $\alpha_{i a} \in k$, and $\alpha_{i, m(i)} \neq 0$. Note that $y_{i}^{-1}=$ $\sum_{b \in J(i)} \beta_{i b} x^{b}$ where $J(i)$ is a well-ordered subset of $\mathbb{Z}^{n}$ with minimum element $-m(i)$, the $\beta_{i b} \in k$, and $\beta_{i,-m(i)}=\alpha_{i, m(i)}^{-1}$.

For any $i, j=1, \ldots, n$, the series $\left\{y_{i}, y_{j}\right\}$ is supported on the set of those $c \in \mathbb{Z}^{n}$ for which $c \geq m(i)+m(j)$ (cf. (35)), and so

$$
\pi\left(\left\{y_{i}, y_{j}\right\}\left(y_{i} y_{j}\right)^{-1}\right)=\left\{x^{m(i)}, x^{m(j)}\right\} x^{-m(i)-m(j)}=m(i) \boldsymbol{\lambda} m(j)^{\operatorname{tr}}
$$

by (33). Thus, $\left(\pi\left(\left\{y_{i}, y_{j}\right\}\left(y_{i} y_{j}\right)^{-1}\right)\right)=A \boldsymbol{\lambda} A^{\text {tr }}$ where $A$ is the matrix in $M_{n}(\mathbb{Z})$ with rows $m(1), \ldots, m(n)$. 
The following corollaries give two immediate applications of Proposition 5.2. They are Poisson analogs of results of Alev and Dumas, who proved that the quotient division ring of a quantum plane $\theta_{q}\left(k^{2}\right)$ cannot be isomorphic to a Weyl skew field [1, Corollaire 3.11(a)], and that for nonroots of unity $q, r \in k^{\times}$, the quotient division rings of $\theta_{q}\left(k^{2}\right)$ and $\theta_{r}\left(k^{2}\right)$ are isomorphic if and only if $q=r^{ \pm 1}[1$, Corollaire 3.11(c)].

Corollary 5.3. - Let $\boldsymbol{\lambda} \in M_{n}(k)$ be antisymmetric. Then $k_{\boldsymbol{\lambda}}\left(x_{1}, \ldots, x_{n}\right)$ is not isomorphic to a Poisson-Weyl field. In fact, it is not isomorphic to any Poisson field containing elements $x$ and $y$ with $\{x, y\}=1$.

Proof. - By Proposition 5.2(a), $\{x, y\} \neq 1$ for all $x, y \in k_{\boldsymbol{\lambda}}\left(x_{1}, \ldots, x_{n}\right)$.

Corollary 5.4. - Let $\boldsymbol{\lambda}=\left[\begin{array}{cc}0 & \lambda \\ -\lambda & 0\end{array}\right]$ and $\boldsymbol{\mu}=\left[\begin{array}{cc}0 & \mu \\ -\mu & 0\end{array}\right]$ for some $\lambda, \mu \in k$. Then $k_{\boldsymbol{\lambda}}\left(x_{1}, x_{2}\right) \cong k_{\boldsymbol{\mu}}\left(x_{1}, x_{2}\right)$ if and only if $\lambda= \pm \mu$.

Proof. - If $\lambda=-\mu$, the $k$-algebra automorphism of $k\left(x_{1}, x_{2}\right)$ fixing $x_{1}$ and sending $x_{2} \mapsto x_{2}^{-1}$ transforms $\{-,-\}_{\boldsymbol{\lambda}}$ to $\{-,-\}_{\boldsymbol{\mu}}$, providing a Poisson isomorphism of $k_{\boldsymbol{\lambda}}\left(x_{1}, x_{2}\right)$ onto $k_{\boldsymbol{\mu}}\left(x_{1}, x_{2}\right)$.

Conversely, assume that $k_{\boldsymbol{\lambda}}\left(x_{1}, x_{2}\right) \cong k_{\boldsymbol{\mu}}\left(x_{1}, x_{2}\right)$. By Proposition 5.2(b),

$$
\left\{A \boldsymbol{\lambda} A^{\text {tr }} \mid A \in M_{n}(\mathbb{Z})\right\}=\left\{B \boldsymbol{\mu} B^{\operatorname{tr}} \mid B \in M_{n}(\mathbb{Z})\right\},
$$

from which we see that $\mathbb{Z} \lambda=\mathbb{Z} \mu$. Since char $k=0$, this implies $\lambda= \pm \mu$.

Cases (b) and (c) of the following theorem are Poisson analogs of results of Panov [30, Theorem 2.19] and Richard [31, Théorème 4.2].

THEOREM 5.5. - Let $\boldsymbol{\lambda}, \boldsymbol{\mu} \in M_{n}(k)$ be antisymmetric, and assume that one of the following holds:

(a) $\boldsymbol{\lambda} \in G L_{n}(k)$.

(b) The subgroup $\sum_{i, j=1}^{n} \mathbb{Z} \lambda_{i j}$ of $(k,+)$ is cyclic.

(c) The subgroup $\sum_{i, j=1}^{n} \mathbb{Z} \lambda_{i j}$ of $(k,+)$ is free abelian of rank $n(n-1) / 2$.

Then $k_{\boldsymbol{\lambda}}\left(x_{1}, \ldots, x_{n}\right) \cong k_{\boldsymbol{\mu}}\left(x_{1}, \ldots, x_{n}\right)$ if and only if there exists $A \in G L_{n}(\mathbb{Z})$ such that $\boldsymbol{\mu}=A \boldsymbol{\lambda} A^{\mathrm{tr}}$.

Proof. - Since the theorem is clear if $n=1$, we may assume that $n \geq 2$. Sufficiency is given by Lemma 5.1. Conversely, assume that $k_{\boldsymbol{\lambda}}\left(x_{1}, \ldots, x_{n}\right) \cong$ $k_{\mu}\left(x_{1}, \ldots, x_{n}\right)$. In view of Proposition 5.2(b), there exist $A, B \in M_{n}(\mathbb{Z})$ such that $\boldsymbol{\mu}=A \boldsymbol{\lambda} A^{\text {tr }}$ and $\boldsymbol{\lambda}=B \boldsymbol{\mu} B^{\operatorname{tr}}$. Note that $\boldsymbol{\lambda}=(B A) \boldsymbol{\lambda}(B A)^{\mathrm{tr}}$.

(a) In this case, it follows from the equation $\boldsymbol{\lambda}=(B A) \boldsymbol{\lambda}(B A)^{\operatorname{tr}}$ that $\operatorname{det}(B A)^{2}=1$, and consequently $A, B \in G L_{n}(\mathbb{Z})$.

(b) By assumption, $\sum_{i, j=1}^{n} \mathbb{Z} \lambda_{i j}=\mathbb{Z} \lambda$ for some $\lambda \in k$. If $\lambda=0$, then $\boldsymbol{\lambda}=\mathbf{0}$ and $\{-,-\}_{\boldsymbol{\lambda}}$ vanishes. In this case, $\{-,-\}_{\boldsymbol{\mu}}$ must also vanish, whence $\boldsymbol{\mu}=\mathbf{0}$ and $\boldsymbol{\mu}=I \boldsymbol{\lambda} I^{\mathrm{tr}}$. 
Now assume that $\lambda \neq 0$. Then $\lambda^{-1} \boldsymbol{\lambda}$ is an antisymmetric integer matrix, and so there exists $C \in G L_{n}(\mathbb{Z})$ such that

$$
C\left(\lambda^{-1} \boldsymbol{\lambda}\right) C^{\mathrm{tr}}=\left[\begin{array}{ccccccc}
0 & d_{1} & 0 & \cdots & & \cdots & 0 \\
-d_{1} & 0 & & & & & \vdots \\
0 & & \ddots & & & & \\
\vdots & & & 0 & d_{r} & & \\
& & & -d_{r} & 0 & & \\
& & & & & 0 & \\
\vdots & & & & & \ddots & \vdots \\
0 & \cdots & & & & \cdots & 0
\end{array}\right]
$$

for some nonzero integers $d_{1}, \ldots, d_{r}$ (e.g., [24, Theorem IV.1]). Hence, we obtain a block matrix decomposition

$$
C \boldsymbol{\lambda} C^{\mathrm{tr}}=\left[\begin{array}{ll}
\Lambda & 0 \\
\mathbf{0} & 0
\end{array}\right]
$$

with $\Lambda \in G L_{2 r}(k)$. Since $C$ is invertible over $\mathbb{Z}$, we may replace $\boldsymbol{\lambda}$ by $C \boldsymbol{\lambda} C^{\mathrm{tr}}$, and so there is no loss of generality in assuming that $\boldsymbol{\lambda}=\left[\begin{array}{ll}\Lambda & 0 \\ 0 & 0\end{array}\right]$.

The equations $\boldsymbol{\mu}=A \boldsymbol{\lambda} A^{\text {tr }}$ and $\boldsymbol{\lambda}=B \boldsymbol{\mu} B^{\text {tr }}$ imply that $\boldsymbol{\lambda}$ and $\boldsymbol{\mu}$ have the same rank, namely $2 r$, and that $\sum_{i, j=1}^{n} \mathbb{Z} \mu_{i j}=\sum_{i, j=1}^{n} \mathbb{Z} \lambda_{i j}=\mathbb{Z} \lambda$. Hence, we also obtain a block matrix decomposition $D \boldsymbol{\mu} D^{\operatorname{tr}}=\left[\begin{array}{cc}M & \mathbf{0} \\ \mathbf{0} & \mathbf{0}\end{array}\right]$ for some $D \in G L_{n}(\mathbb{Z})$ and some $M \in G L_{2 r}(k)$. As above, there is no loss of generality in assuming that $\boldsymbol{\mu}=\left[\begin{array}{cc}M & \mathbf{0} \\ \mathbf{0} & \mathbf{0}\end{array}\right]$.

Write $A$ and $B$ in block form as

$$
A=\left[\begin{array}{ll}
A_{11} & A_{12} \\
A_{21} & A_{22}
\end{array}\right] \quad B=\left[\begin{array}{ll}
B_{11} & B_{12} \\
B_{21} & B_{22}
\end{array}\right]
$$

where $A_{11}$ and $B_{11}$ are $2 r \times 2 r$. The equations $\boldsymbol{\mu}=A \boldsymbol{\lambda} A^{\operatorname{tr}}$ and $\boldsymbol{\lambda}=B \boldsymbol{\mu} B^{\operatorname{tr}}$ now say that

$$
\left[\begin{array}{cc}
M & \mathbf{0} \\
\mathbf{0} & \mathbf{0}
\end{array}\right]=\left[\begin{array}{ll}
A_{11} L A_{11}^{\mathrm{tr}} & A_{11} L A_{21}^{\mathrm{tr}} \\
A_{21} L A_{11}^{\mathrm{tr}} & A_{21} L A_{21}^{\mathrm{tr}}
\end{array}\right] \quad\left[\begin{array}{ll}
\Lambda & \mathbf{0} \\
\mathbf{0} & \mathbf{0}
\end{array}\right]=\left[\begin{array}{ll}
B_{11} M B_{11}^{\mathrm{tr}} & B_{11} M B_{21}^{\mathrm{tr}} \\
B_{21} M B_{11}^{\mathrm{tr}} & B_{21} M B_{21}^{\mathrm{tr}}
\end{array}\right],
$$


and so $\Lambda=\left(B_{11} A_{11}\right) \Lambda\left(B_{11} A_{11}\right)^{\mathrm{tr}}$. As in case (a), it follows that $A_{11} \in$ $G L_{2 r}(\mathbb{Z})$. Hence, the matrix $E=\left[\begin{array}{cc}A_{11} & 0 \\ 0 & I_{n-2 r}\end{array}\right]$ lies in $G L_{n}(\mathbb{Z})$. Since $\boldsymbol{\mu}=$ $E \boldsymbol{\lambda} E^{\mathrm{tr}}$, the proof of part (b) is complete.

(c) Since $\boldsymbol{\lambda}$ is antisymmetric, the group $\sum_{i, j=1}^{n} \mathbb{Z} \lambda_{i j}$ is generated by the $\lambda_{i j}$ for $i<j$, so the assumption of rank $n(n-1) / 2$ implies that $\left\{\lambda_{i j} \mid 1 \leq i<j \leq n\right\}$ is a basis for $\sum_{i, j=1}^{n} \mathbb{Z} \lambda_{i j}$. As noted in the proof of part (b), $\sum_{i, j=1}^{n} \mathbb{Z} \mu_{i j}=$ $\sum_{i, j=1}^{n} \mathbb{Z} \lambda_{i j}$, and so this group also has a basis $\left\{\mu_{i j} \mid 1 \leq i<j \leq n\right\}$.

Next, identify $\boldsymbol{\lambda}$ with the linear transformation on $k^{n}$ given by left multiplication of $\boldsymbol{\lambda}$ on column vectors. We claim that $\mathbb{Z}^{n} \cap \operatorname{ker} \boldsymbol{\lambda}=\{\mathbf{0}\}$. If $a=\left(a_{1}, \ldots, a_{n}\right)^{\operatorname{tr}} \in \mathbb{Z}^{n} \cap \operatorname{ker} \boldsymbol{\lambda}$, then $\lambda_{12} a_{2}+\lambda_{13} a_{3}+\cdots+\lambda_{1 n} a_{n}=0$. Since $\lambda_{12}, \ldots, \lambda_{1 n}$ are $\mathbb{Z}$-linearly independent, it follows that $a_{2}=a_{3}=\cdots=a_{n}=0$. Then $\lambda_{21} a_{1}=0$, which implies $a_{1}=0$ because $\lambda_{21}=-\lambda_{12} \neq 0$. Thus $a=\mathbf{0}$, establishing the claim. Since $\boldsymbol{\lambda}=(B A) \boldsymbol{\lambda}(B A)^{\operatorname{tr}}$, it follows that $\mathbb{Z}^{n} \cap \operatorname{ker}(B A)^{\operatorname{tr}}=$ $\{\mathbf{0}\}$. But $(B A)^{\text {tr }}$ is an integer matrix, so we obtain $\operatorname{det}(B A)^{\operatorname{tr}} \neq 0$, and thus $\operatorname{det}(B A) \neq 0$.

Write $B A=\left(d_{i j}\right)$, and compare entries in the equation $\boldsymbol{\lambda}=(B A) \boldsymbol{\lambda}(B A)^{\operatorname{tr}}$ :

$$
\lambda_{i j}=\sum_{l, m=1}^{n} d_{i l} \lambda_{l m} d_{j m}=\sum_{1 \leq l<m \leq n}\left(d_{i l} d_{j m}-d_{i m} d_{j l}\right) \lambda_{l m}
$$

for all $i, j$. Since the $\lambda_{l m}$ for $l<m$ are $\mathbb{Z}$-linearly independent, we find that

$$
d_{i l} d_{j m}-d_{i m} d_{j l}=\delta_{i l} \delta_{j m}
$$

for $1 \leq i<j \leq n$ and $1 \leq l<m \leq n$. It follows from the Laplace relations that all the $2 \times 2$ and larger minors of $B A$ for which the row and column index sets differ must vanish. In particular, this implies that the adjoint matrix $D=\operatorname{adj}(B A)$ is diagonal. Since $B A D=\operatorname{det}(B A) I_{n}$ and $\operatorname{det}(B A) \neq 0$, we conclude that $B A$ must be a diagonal matrix.

The equation $\boldsymbol{\lambda}=(B A) \boldsymbol{\lambda}(B A)^{\mathrm{tr}}$ now reduces to $\lambda_{i j}=d_{i i} \lambda_{i j} d_{j j}$ for all $i, j$, whence $d_{i i} d_{j j}=1$ for all $i<j$. Since $n \geq 2$ and the $d_{i i}$ are integers, $d_{i i}= \pm 1$ for all $i$, whence $B A \in G L_{n}(\mathbb{Z})$. Therefore $A \in G L_{n}(\mathbb{Z})$, proving part (c).

It is tempting to conjecture that the equivalence of Theorem 5.5 holds for arbitrary antisymmetric $\boldsymbol{\lambda}, \boldsymbol{\mu} \in M_{n}(k)$.

Acknowledgements. - We thank J. Alev for extensive discussions on Poisson algebras, and T. Levasseur for helpful correspondence and references concerning differentials of group actions.

TOME $139-2011-\mathrm{N}^{\mathrm{O}} 1$ 


\section{BIBLIOGRAPHY}

[1] J. Alev \& F. Dumas - "Sur le corps des fractions de certaines algèbres quantiques", J. Algebra 170 (1994), p. 229-265.

[2] K. A. Brown \& K. R. GoodeARL - Lectures on algebraic quantum groups, Advanced Courses in Mathematics. CRM Barcelona, Birkhäuser, 2002.

[3] K. A. Brown \& I. Gordon - "Poisson orders, symplectic reflection algebras and representation theory", J. reine angew. Math. 559 (2003), p. $193-216$.

[4] G. Cauchon - "Effacement des dérivations et spectres premiers des algèbres quantiques", J. Algebra 260 (2003), p. 476-518.

[5] P. M. CoHN - Universal algebra, second ed., Mathematics and its Applications, vol. 6, D. Reidel Publishing Co., 1981.

[6] W. Dicks \& J. LEWIN - "A Jacobian conjecture for free associative algebras", Comm. Algebra 10 (1982), p. 1285-1306.

[7] J. Dixmier - "Idéaux primitifs dans les algèbres enveloppantes", J. Algebra 48 (1977), p. 96-112.

[8] K. R. Goodearl - "A Dixmier-Moeglin equivalence for Poisson algebras with torus actions", in Algebra and its applications, Contemp. Math., vol. 419, Amer. Math. Soc., 2006, p. 131-154.

[9] K. R. Goodearl \& E. S. Letzter - "Prime factor algebras of the coordinate ring of quantum matrices", Proc. Amer. Math. Soc. 121 (1994), p. 1017-1025.

[10] _ , "The Dixmier-Moeglin equivalence in quantum coordinate rings and quantized Weyl algebras", Trans. Amer. Math. Soc. 352 (2000), p. $1381-1403$.

[11] K. R. Goodearl \& M. YAKImov - "Poisson structures on affine spaces and flag varieties. II", Trans. Amer. Math. Soc. 361 (2009), p. 5753-5780.

[12] T. J. Hodges \& T. Levasseur - "Primitive ideals of $\mathbf{C}_{q}[\mathrm{SL}(3)]$ ", Comm. Math. Phys. 156 (1993), p. 581-605.

[13] _ "Primitive ideals of $\mathbf{C}_{q}[\mathrm{SL}(n)]$ ", J. Algebra 168 (1994), p. 455-468.

[14] T. J. Hodges, T. Levasseur \& M. Toro - "Algebraic structure of multiparameter quantum groups", Adv. Math. 126 (1997), p. 52-92.

[15] K. L. HoRTON - "The prime and primitive spectra of multiparameter quantum symplectic and Euclidean spaces", Comm. Algebra 31 (2003), p. $4713-4743$.

[16] R. S. IRving \& L. W. SMALL - "On the characterization of primitive ideals in enveloping algebras", Math. Z. 173 (1980), p. 217-221.

[17] A. JosEPH - "On the prime and primitive spectra of the algebra of functions on a quantum group", J. Algebra 169 (1994), p. 441-511. 
[18] _ Quantum groups and their primitive ideals, Ergebnisse Math. Grenzg., vol. 29, Springer, 1995.

[19] A. KAMITA - "Quantum deformations of certain prehomogeneous vector spaces. III", Hiroshima Math. J. 30 (2000), p. 79-115.

[20] B. Kostant \& N. WALlach - "Gelfand-Zeitlin theory from the perspective of classical mechanics. II", in The unity of mathematics, Progr. Math., vol. 244, Birkhäuser, 2006, p. 387-420.

[21] F. Loose - "Symplectic algebras and Poisson algebras", Comm. Algebra 21 (1993), p. 2395-2416.

[22] C. Moeglin - "Idéaux primitifs des algèbres enveloppantes", J. Math. Pures Appl. 59 (1980), p. 265-336.

[23] I. M. Musson - "Ring-theoretic properties of the coordinate rings of quantum symplectic and Euclidean space", in Ring theory (Granville, OH, 1992), World Sci. Publ., River Edge, NJ, 1993, p. 248-258.

[24] M. Newman - Integral matrices, Academic Press, 1972, Pure and Applied Mathematics, Vol. 45.

[25] M. Noumi - "Macdonald's symmetric polynomials as zonal spherical functions on some quantum homogeneous spaces", Adv. Math. 123 (1996), p. $16-77$.

[26] S.-Q. OH - "Catenarity in a class of iterated skew polynomial rings", Comm. Algebra 25 (1997), p. 37-49.

[27] _ "Symplectic ideals of Poisson algebras and the Poisson structure associated to quantum matrices", Comm. Algebra 27 (1999), p. 2163-2180.

[28] _ _ "Poisson polynomial rings", Comm. Algebra 34 (2006), p. 12651277.

[29] _ "Quantum and Poisson structures of multi-parameter symplectic and Euclidean spaces", J. Algebra 319 (2008), p. 4485-4535.

[30] A. N. PAnov - "Skew field of rational functions on $\mathrm{GL}_{q}(n, K)$ ", Funktsional. Anal. i Prilozhen. 28 (1994), p. 75-77.

[31] L. RiCHARD - "Sur les endomorphismes des tores quantiques", Comm. Algebra 30 (2002), p. 5283-5306.

[32] E. STRICKLAND - "Classical invariant theory for the quantum symplectic group", Adv. Math. 123 (1996), p. 78-90.

[33] P. TAuvel \& R. W. T. YU - Lie algebras and algebraic groups, Springer Monographs in Math., Springer, 2005.

[34] _ _ "Algèbres de Poisson et algèbres de Lie résolubles", Comm. Algebra 38 (2010), p. 2317-2353.

[35] M. VANCLIFF - "Primitive and Poisson spectra of twists of polynomial rings", Algebr. Represent. Theory 2 (1999), p. 269-285. 
[36] M. Vergne - "La structure de Poisson sur l'algèbre symétrique d'une algèbre de Lie nilpotente", Bull. Soc. Math. France 100 (1972), p. 301335 . 